\title{
HOLOMORPHIC FIBER BUNDLES OVER RIEMANN SURFACES
}

\author{
HELMUT RÖHRL
}

For the purpose of this paper a fiber bundle $F \rightarrow X$ over a Riemann surface $X$ is meant to be a fiber bundle in the sense of N. Steenrod [62] where the base space is $X$, the fiber a complex space, the structure group $G$ a complex Lie group that acts as a complex transformation group on the fiber, and the transition functions $g_{i j}(x)$ are holomorphic mappings into $G$. Correspondingly, cross-sections are assumed to be holomorphic cross-sections. We shall use freely the notations of [62]. Whenever we report about families of fiber bundles we mean holomorphic families of fiber bundles; the basic notations concerning families of fiber bundles can be found in [30] and shall also be used freely. Triviality of bundles resp. families of bundles is always supposed to be holomorphic triviality.

1. Classification of fiber bundles and reduction of the structure group. The classification of fiber bundles over noncompact Riemann surfaces offers no problem on account of

Theorem $1.0[15 ; 54]$. Every fiber bundle over a noncompact Riemann surface is trivial, provided the structure group $G$ is connected. ${ }^{1}$

For compact Riemann surfaces, however, the situation is entirely different. In the general case it seems to be quite difficult to give a classification. Yet one has some results if either the fiber and the structure group or else the base space is sufficiently special.

For the rest of this section $X$ shall always denote a compact Riemann surface unless stated otherwise.

There is a preliminary result concerning line bundles $L \rightarrow X$, i.e. fiber bundles with fiber the complex line $C^{1}$ and structure group the multiplicative group $G L(1, C)$ of complex numbers acting upon $C^{1}$ in the usual way, a result that first has been proved in a much more

An address delivered before the Milwaukee meeting of the Society on November 18,1961 , by invitation of the Committee to Select Hour Speakers for Western Sectional Meetings; received by the editors January 2, 1962.

1 If $G$ is not connected, the theorem fails to be true as can be seen from the following example: Let $X$ be the complex plane without the origin, $G$ the multiplicative group of $n$th roots of unity acting on the unit circle by (left) multiplication, and the fiber bundle be defined by a covering of $X$, consisting of two domains $U_{1}, U_{2}$ bounded by straight lines through the origin, and the transition function that equals 1 in one component of $\left.U_{1}\right\urcorner U_{2}$ and $e^{2 \pi i / n}$ in the other component of $U_{1} \cap U_{2}$. 
general setting $([29]$, for an elementary proof in our case see [54; 56]), namely

Proposition 1.1. The set of isomorphy classes of line bundles $L \rightarrow X$ is in a bijective correspondence with the set of divisor classes of $X$.

Let us consider vector bundles $W \rightarrow X$ of $r a n k r$, i.e. fiber bundles with fiber the complex number space $C^{r}$ of dimension $r$ and structure group the group $G L(r, C)$ of complex $r \times r$-matrices acting upon $C^{r}$ in the usual way. A nonzero vector bundle is called decomposable if it is the Whitney sum of two nonzero vector bundles; otherwise it is called indecomposable. A Remak decomposition of a vector bundle is a decomposition into a Whitney sum of indecomposable vector bundles. Then we get

THEOREM 1.2 [5]. Every vector bundle over a compact Riemann surface admits a Remak decomposition. This decomposition is uniquely determined up to rearrangement of the summands in the decomposition.

Theorem 1.2 shows that it is sufficient to classify indecomposable vector bundles in order to achieve a classification of vector bundles. This classification can be carried out in case $X$ is the Riemann sphere $P^{1}$. The result is

TheOREM $1.3[7 ; 18]$. The line bundles are the only indecomposable vector bundles over $P^{1}$.

On account of Theorem 1.2 the statement of Theorm 1.3 is equivalent to: every vector bundle over $P^{1}$ is the Whitney sum of line bundles. Thus the classification of vector bundles over $P^{1}$ is completed if we have classified line bundles over $P^{1}$. For that purpose we choose a nonconstant, single valued, meromorphic function $x(Q)$ on $P^{1}$. Then $U_{0}=\{Q \mid x(Q) \neq \infty\}, U_{\infty}=\{Q \mid x(Q) \neq 0\}$ constitute an open covering of $P^{1}$. Denoting the line bundle defined by the transition function $g_{0, \infty}(Q)=x(Q)$ by $L_{1} \rightarrow P^{1}$, an easy argument involving Proposition 1.1 leads to

Proposition 1.4. For every line bundle $L \rightarrow P^{1}$ there is a (uniquely determined) integer $d$ such that $L$ is isomorphic to the Whitney product $L_{1}^{d}$ of $d$ copies of $L_{1}$.

From this one derives

PROPOSITION 1.5 [18]. The structure group of a vector bundle $W \rightarrow P^{1}$ can be reduced to the complex orthogonal group if and only if $W$ is isomorphic to its dual bundle $W^{*} \rightarrow P^{1}$. 
Theorem 1.3 together with Proposition 1.4 state that the isomorphy classes of vector bundles of rank $r$ over $P^{1}$ are in a bijective correspondence with the set of $r$-tuples of integers $\left(k_{1}, \cdots, k_{r}\right)$ fulfilling $k_{1} \leqq k_{2} \leqq \cdots \cdots k_{r}$. This classification in turn gives rise to a complete classification of principal $P G L(r, C)$-bundles where $P G L(r, C)$ is the complex projective group corresponding to $G L(r+1, C)$. Indeed, from the exact sequence of groups

$$
0 \rightarrow G L(1, C) \rightarrow G L(r+1, C) \rightarrow P G L(r, C) \rightarrow 0
$$

we get a surjective mapping ${ }^{2}$

$$
H^{1}\left(X, G L(r+1, C)_{\omega}\right) \rightarrow H^{1}\left(X, P G L(r, C)_{\omega}\right) .
$$

Thus our last remark shows (cf. also [23])

Proposition 1.6. The set of isomorphy classes of principal PGL $r, C)$ bundles over $P^{1}$ is in a bijective correspondence with the set of $r$-tuples of non-negative integers $\left(k_{1}, \cdots, k_{r}\right)$ fulfilling $k_{1} \leqq k_{2} \leqq \cdots \leqq k_{r}$.

In case the structure group of a fiber bundle over $P^{1}$ is not of as simple a structure as the ones dealt with so far, we cannot expect the results to be as smooth. Yet one has

TheORem 1.7 [18]. Let $F \rightarrow P^{1}$ be a fiber bundle whose structure group $G$ is reductive. Then $F \rightarrow P^{1}$ admits a reduction to the Cartan subgroup of $G$; this is unique up to an action of the Weyl group of $G$.

So far the results have pertained to fiber bundles over the Riemannian sphere. Now we shall turn to another case in which we can get ample information, the case in which the base space $X$ is a torus $T^{1}$, i.e. a compact Riemann surface of genus 1 . In order to be able to phrase the statements we need a few definitions.

Let $W \rightarrow X$ be a vector bundle. Then we can associate with it a line bundle det $W \rightarrow X$ by switching from the transition functions $g_{i j}$ defining $W$ to the transition functions det $g_{i j}$. Furthermore, let $c_{1}(W)$ be the first Chern class of $W$ and $\mu \in H^{2}(X, Z)$ the fundamental class. Then there is an integer $d$ such that $d \cdot \mu=c_{1}(W) . d$ is called the degree of $W$ and denoted by $\operatorname{deg}(W)$. It is easy to see that $\operatorname{deg}(W)$ $=\operatorname{deg}(\operatorname{det} W)$. Given a line bundle $L \rightarrow X$ and a meromorphic section $s$ in $L, s$ defines in an obvious manner a divisor $\operatorname{div} s$ and we have the relation $\operatorname{deg}(\operatorname{div} s)=\operatorname{deg}(L)$ where $\operatorname{deg}(\operatorname{div} s)$ equals the number of zeros of $s$ minus the number of poles of $s$, both counted according to

${ }^{2}$ For a complex Lie group $G, G_{\omega}$ denotes the sheaf of germs of holomorphic mappings (of a given complex space $X$ ) into $G ; H^{1}\left(X, G_{\omega}\right)$ is the first cohomology set of $X$ with values in $G_{\omega}$. 
their multiplicities. It can be shown easily that the integer $d$ occurring in Proposition 1.4 is actually equal to $\operatorname{deg}(L)$.

Next we denote by $\&(X)$ the set of all isomorphy classes of vector bundles over $X$ and by $\mathbb{E}(X ; r, d)$ the subset of $\mathbb{E}(X)$ corresponding to indecomposable vector bundles of rank $r$ and degree $d$. With these notations follows

Proposition 1.8. Let $L$ be a line bundle of degree 1 over $X$. Then the mapping that sends the vector bundle $E$ into $E \otimes L^{n}$ determines a bijective mapping $\mathbb{E}(X ; r, d) \rightarrow \mathbb{E}(X ; r, d+n r)$.

This shows that we may restrict ourselves to the discussion of $\&(X ; r, d)$ where $0 \leqq d<r$ holds.

THEOREM 1.9 [3]. There exists exactly one isomorphy class in $\mathfrak{E}\left(T^{1} ; r, 0\right)$ that contains a vector bundle $W_{r}$ admitting a nontrivial holomorphic section. The mapping $L \rightarrow W_{r} \otimes L$ of line bundles into vector bundles or rank $r$ gives rise to a bijective mapping $\mathbb{E}\left(T^{1} ; 1,0\right) \rightarrow \mathbb{S}\left(T^{1} ; r, 0\right)$. In particular, there is a natural isomorphism between $W$ and $W_{r} \otimes \operatorname{det} W$, $r$ being the rank of the vector bundle $W$ of degree 0 .

Having chosen a line bundle $L$ of degree 1 we now want to define by induction a mapping $\alpha_{r, d}: \&\left(T^{1} ; h, 0\right) \rightarrow \&\left(T^{1} ; r, d\right)$ where $h$ is the greatest common divisor of $r$ and $d$. For that purpose we define the mapping $\bar{\alpha}_{r, d}$ for vector bundles of rank $h$ and degree 0 inductively by

$$
\begin{aligned}
\bar{\alpha}_{r, 0}(W) & =W, \\
\bar{\alpha}_{r, r+d}(W) & =\bar{\alpha}_{r, d}(W) \otimes L,
\end{aligned}
$$

(iii) 1 denoting the trivial line bundle over $T^{1}$ there is an exact sequence

$$
0 \rightarrow 1^{d} \rightarrow \bar{\alpha}_{r, d}(W) \rightarrow \bar{\alpha}_{r-d, d}(W) \rightarrow 0
$$

provided that $0<d<r$ holds.

It can be shown [3] that by passing to isomorphy classes these conditions determine the mappings $\alpha_{r, d}$ uniquely and that there are mappings fulfilling the conditions (i)-(iii). Furthermore

Theorem $1.10[3] . \alpha_{r, d}: \&\left(T^{1} ; h, 0\right) \rightarrow \&\left(T^{1} ; r, d\right)$ is bijective.

Therefore we have bijective mappings $\mathbb{E}\left(T^{1} ; r, d\right) \rightarrow \mathfrak{F}\left(T^{1} ; 1,0\right)$. But $\mathbb{E}\left(T^{1} ; 1,0\right)$ can be easily seen to be in a bijective correspondence with $T^{1}$ itself. This finally gives rise to a bijective mapping $\beta_{r, d}: \mathbb{E}\left(T^{1} ; r, d\right) \rightarrow T^{1}$, for which the formula

$$
h \cdot \beta_{r, d}(W)=\beta_{1, d}(\operatorname{det} W)
$$

holds, where $h$ is again the greatest common divisor of $r$ and $d$ and 
$h \cdot x$ equals $x+x+\cdots+x$ ( $h$ times), the addition being the one in the abelian group $T^{1}$.

Theorem 1.9, Theorem 1.10, and our last remark describe completely the additive structure of $\mathbb{E}\left(T^{1}\right)$ induced by the Whitney sum. It is equally interesting to determine the multiplicative structure of $\mathbb{E}\left(T^{1}\right)$ induced by the Whitney product. This is carried out in [3] but shall be omitted here.

Since for a line bundle $L$ both $W$ and $W \otimes L$ decompose simultaneously, we may speak of a principal $P G L(r, C)$-bundle as being indecomposable. Then we derive from a more detailed study of Theorem 1.9

PROPOSITION 1.11 [3]. There are exactly $r+1$ isomorphy classes of indecomposable principal $P G L(r, C)$-bundles over $T^{1}$.

In the case of $P^{1}$ resp. $T^{1}$ as base space we got reasonably good information concerning isomorphy classes, due to the fact that every divisor of degree 0 on $P^{1}$ is a principal divisor resp. that the canonical divisor on $T^{1}$ is a principal divisor. Therefore, we cannot expect to get equally good results if the base space $X$ has genus $\geqq 2$. Yet it can be shown that the structure group of a vector bundle can be reduced:

Proposition 1.12 [3; 60]. Let $W \rightarrow X$ be a vector bundle of rank $r$. Then the structure group can be reduced to the group $\Delta(r, C)$ of triangular $r \times r$-matrices.

From another method of reducing the structure group of vector bundles, one can conclude that $\mathbb{E}(X ; r, d)$ is bijectively mapped onto $F / R$ where $F$ is a certain vector bundle over the Picard variety of $X$ and $R$ is a fiber preserving equivalence relation in $F[3 ; 26]$. However, it seems to be difficult to obtain more insight into the nature of the equivalence relation $R$, except in special cases [2].

Now we shall deal with fiber bundles whose group is the affine group $G A(r, C)$ in dimension $r$ over the complex number field. There we have an exact sequence of groups

$$
0 \rightarrow C^{r} \rightarrow G A(r, C) \stackrel{\pi}{\rightarrow} G L(r, C) \rightarrow 0
$$

which induces a surjective mapping

$$
\pi: H^{1}\left(X, G A(r, C)_{\omega}\right) \rightarrow H^{1}\left(X, G L(r, C)_{\omega}\right) .
$$

In order to achieve a classification of principal $G A(r, C)$-bundles it is necessary to determine $\pi^{-1}(\xi)$ for a given element $\xi \in H^{1}\left(X, G L(r, C)_{\omega}\right)$. There we have

Proposition $1.13[2 ; 45 ; 66]$. Given $\xi \in H^{1}\left(X, G L(1, C)_{\omega}\right)$, the set 
$\pi^{-1}(\xi)$ consists of $\xi$ itself together with a complex projective space $P^{k}$ whose dimension $k$ equals $\operatorname{dim}_{C} H^{1}(X, \mathfrak{D}(\xi))-1 .^{3}$

Corollary. Let $X$ be the Riemannian sphere. Then the dimension $k$ of the complex projective space mentioned in Proposition 1.13 equals $\min (0,-1-\operatorname{deg}(\xi))-1$.

Corollary. Let $g$ be the genus of $X$. Then $\pi^{-1}(\xi)$ consists of $\xi$ alone, provided $\operatorname{deg}(\xi)>2 g-2$.

Hence we have reached a classification of principal $G A(1, C)$-bundles over a compact Riemann surface, on account of Proposition 1.1. After having classified the principal $G A(1, C)$-bundles one can attempt a classification of principal $P G L(1, C)$-bundles. For that purpose let $j: G A(1, C) \rightarrow P G L(1, C)$ be the canonical injection. Then one can prove by using results of $[27]$.

Proposition 1.14 [2]. The mapping

$$
H^{1}\left(X, G A(1, C)_{\omega}\right) \rightarrow H^{1}\left(X, P G L(1, C)_{\omega}\right)
$$

which is induced by $j$ is surjective.

Having already classified the principal $G A(1, C)$-bundles, this means that the classification of principal $P G L(1, C)$-bundles is finished as soon as we have a criterion telling whether two principal $G A(1, C)$-bundles define the same principal $P G L(1, C)$-bundle. Such a criterion can indeed be found [2]. Unfortunately, it is difficult to decide for given principal $G A(1, C)$-bundles whether they fulfill the conditions of this criterion. If the genus of $X$ does not exceed 2 the calculations involved have been carried out explicitly in [2] and lead to the desired classification. Contrary to Proposition 1.11 this classification is not restricted to indecomposable bundles and therefore not even for $X=T^{1}$ contained in Proposition 1.11. In this latter case we get

Proposition 1.15 [2]. Every principal PGL(1,C)-bundle over $T^{1}$ is isomorphic either to a $G L(1, C)$-bundle of non-negative degree or else to one of certain two $G A(1, C)$-bundles (with $P G L(1, C)$ as fiber).

In case the genus of $X$ equals 2 the situation is more complicated [2] and its description may be omitted here.

A question that is closely related to the reduction of the structure group is the following. Given a complex Lie group $G$, the constant sheaf $G$ over $X$ is a subsheaf of the sheaf $G_{\omega}$. A fiber bundle $F \rightarrow X$ with structure group $G$ is given by an element in $H^{1}\left(X, G_{\omega}\right)$. Under

${ }^{3} \mathfrak{O}(\xi)$ denotes the sheaf of germs of holomorphic sectons in the line bundle defined by $\xi$. 
which conditions can $F \rightarrow X$ be defined by an element in $H^{1}(X, G)$ ? In other words, when is it possible to introduce in $F$ fiber coordinates in such a way that the new transition functions are constant? Immediately a class of fiber bundles can be given that has the required property. Let $\mu: \pi_{1}(X) \rightarrow G$ be a homomorphism of the fundamental group of $X$ into $G$. The universal covering $\tilde{X}$ of $X$ is a principal $\pi_{1}(X)$-bundle over $X$ and hence the homomorphism $\mu$ induces a principal $G$-bundle $\xi_{\mu} \rightarrow X$ (for different descriptions see $[13 ; 54]$ ). Because $\pi_{1}(X)$ is discrete $\xi_{\mu}$ has constant transition functions with respect to suitable fiber coordinates. $\xi_{\mu} \rightarrow X$ is said to arise from the homomorphism $\mu$. With these definitions we get

THEOREM 1.16 [4]. The following statements concerning principal Gbundles $P$ over $X$ are equivalent:

(i) $P$ arises from a homomorphism $\mu: \pi_{1}(X) \rightarrow G$,

(ii) $P$ is defined by an element of $H^{1}(X, G)$,

(iii) $P$ has a holomorphic connection,

(iv) $P$ has a discrete form $P^{\#}$.

The latter statement means: denoting the abstract group corresponding to $G$ and being equipped with the discrete topology by $G^{\#}, P^{\#}$ is a (topological) principal G\#-bundle over $X$ such that there is a bijective and continuous bundle mapping $P^{H} \rightarrow P$.

As an immediate consequence of Theorem 1.16 we get that a principal bundle over $P^{1}$ admits a holomorphic connection if and only if it is trivial.

Theorem 1.16 together with some other results lead to criteria concerning indecomposability of vector bundles $W \rightarrow X$. There we have among others

Proposition 1.17 [4]. $W \rightarrow X$ is an indecomposable vector bundle if and only if the C-algebra $H^{0}(X, \mathfrak{O}(\operatorname{Hom}(W, W)))$ has the properties ${ }^{4}$

(i) $H^{0}(X, \mathfrak{D}(\operatorname{Hom}(W, W)))$ has a unit element e,

(ii) the nilpotent elements of $\left.H^{0}(X, \mathfrak{D}) \operatorname{Hom}(W, W)\right)$ ) form a subalgebra $N$,

(iii) as a $C$-vector space $H^{0}(X, \mathfrak{O}(\operatorname{Hom}(W, W))$ can be written as $\{e\} \oplus N$ where $\{e\}$ is the subspace generated by $e$.

And finally

TheOREM $1.16[4 ; 65]$. Let $W_{1} \oplus \cdots \oplus W_{k}$ be a Remak decomposition of $W \rightarrow X$. Then $W$ arises from a homomorphism of the fundamental

${ }^{4} \operatorname{Hom}(W, W)$ is defined as the vector bundle belonging to the locally free sheaf $\operatorname{Hom}(\mathfrak{O}(W), \mathfrak{O}(W))$ where $\mathfrak{O}(W)$ is the sheaf of germs of holomorphic sections in $W$. 
group into $G L(r, C)$ if and only if $\operatorname{deg}\left(W_{\kappa}\right)=0$ for $\kappa=1, \cdots, k$.

Using the results and techniques of [30], one is able to classify those principal $G A(1, C)$-bundles that arise from a homomorphism of the fundamental group:

THEOREM 1.19 [46]. Let $X$ be a compact Riemann surface of genus $g \geqq 2, J$ the Jacobian variety of $X$, and $J^{\prime}=J-\{0\}$. Then there is a fiber bundle $M \rightarrow J^{\prime}$ with fiber the $(g-2)$-dimensional complex projective space and a bijective mapping $j$ of $M$ onto the set of isomorphy classes of those indecomposable principal $G A(1, C)$-bundles that arise from homomorphisms of the fundamental group of $X$ and whose image under the mapping (1) is not trivial. Furthermore, there is a holomorphic family $\mathfrak{F} \rightarrow \mathfrak{B} \rightarrow M$ of principal $G A(1, C)$-bundles over $X$ such that for every $t \in M$ the restriction $F_{t} \rightarrow V_{t}$ has the above properties and corresponds to $t$ under the mapping $j$.

In this context it should be remarked that a very detailed study of those vector bundles over $T^{1}$ that arise from a homomorphism of the fundamental group can be found in [39].

Now we shall bring some results concerning families of fiber bundles $\mathfrak{F} \rightarrow \mathfrak{B} \rightarrow M$ over Riemann surfaces whose parameter space is assumed to be connected. The family $\mathfrak{B} \rightarrow M$ of base spaces is assumed to be given once and for ever. Again we deal first with families whose family of base spaces is a family of noncompact Riemann surfaces.

Let $X_{0}$ be a noncompact Riemann surface and suppose that $\mathfrak{B} \rightarrow U_{0}$ is a holomorphic family $\subseteq$ of complex structures on $X_{0}$ whose parameter space $U_{0}$ is a polycylinder. In particular, $\mathfrak{B} \rightarrow U_{0}$ is a differentiable fiber bundle with fiber $X_{0}$ that is differentiably trivial, i.e. differentiably isomorphic to $U_{0} \times X_{0}$. Choosing a relatively compact and open subset $X$ of $X_{0}$ and an open subset $U$ of $U_{0}$ we can form the subset $U \times X$ of $U_{0} \times X_{0}$. It corresponds to an open subset of $\mathfrak{B}$ which, equipped with the induced complex structure, is a complex manifold $\mathfrak{S}(U, X)$. Under these circumstances we have

Proposition 1.20 [47]. For any point $t \in U_{0}$ there is a neighborhood $U$ such that $\mathfrak{S}(U, X)$ is holomorphically complete.

Choosing for $U$ a polycylinder we see that $H_{q}(U \times X, Z)=\{0\}$ for $q \geqq 2$. Hence every topological fiber bundle $F \rightarrow U \times X$ is topologically trivial provided the structure group is connected. Therefore [15] implies

THEOREM 1.21. Let $\mathfrak{B} \rightarrow U_{0}$ be a holomorphic family $\mathfrak{S}$ of complex structures on the noncompact Riemann surface $X_{0}$ whose parameter 
space is a polycylinder. Let $X$ be a relatively compact and open subset of

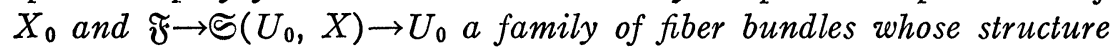
group is connected. Then for every $t \in U_{0}$ there is a neighborhood $U$ such that the restriction $\mathfrak{F} \mid \mathfrak{S}(U, X) \rightarrow \Im(U, X)$ is trivial.

In general, however, a holomorphic family $\mathfrak{B} \rightarrow M$ of complex structures on $X_{0}$ is, differentiably speaking, a fiber bundle that is defined by means of a certain structure group $H$. If every element of $H$ happens to map $X$ onto itself, then we get in an obvious way again a complex manifold $\mathfrak{S}(M, X)$. Now suppose that we are given a family $\mathfrak{F} \rightarrow \mathfrak{S}(M, X) \rightarrow M$ of fiber bundles. Then Theorem 1.21 states a certain local triviality of this family. If we choose an open covering of $M$ consisting of sufficiently small sets $U_{i}, i \in I$, then we can reconstruct the global family from the family of base spaces $\mathfrak{S}(M, X) \rightarrow M$ and the restrictions $\mathfrak{F} \mid \subseteq\left(U_{i}, X\right) \rightarrow \subseteq\left(U_{i}, X\right)$ provided we know in which way we have to match these restrictions. That means that the family defines in a unique way an element in the first cohomology set of $M$ with values in the sheaf of germs of those fiber preserving automorphisms of $\mathfrak{F} \rightarrow \mathfrak{S}(M, X)$ that induce the identity mapping on $\mathfrak{S}(M, X)$. This sheaf obviously depends only on the family $\subseteq(M, X) \rightarrow M$ of base spaces, the structure group $G$ of the family of fiber spaces, and the fiber $F$ of this family. Denoting it by $\operatorname{Aut}(\subseteq(M, X), F, G)$ we get the

CoRollary. The set of isomorphy classes of families $\mathfrak{F} \rightarrow \mathfrak{S}(M, X)$ $\rightarrow M$ of fiber bundles whose parameter space $M$ is a complex manifold corresponds bijectively to the set $H^{1}(M, \operatorname{Aut}(\Im(M, X), F, G))$.

Due to the restrictions imposed on $X$ Theorem 1.21 is not quite satisfactory. However, in case that the family $\mathfrak{B} \rightarrow M$ is locally trivial, i.e. a (holomorphic and not only differentiable) fiber bundle Theorem 1.21 and its Corollary hold also for $X=X_{0}$.

Finally we shall deal with families of fiber bundles over the Riemannian sphere $P^{1}$ in which case we only require that the parameter space is a complex space. Then we get

THEOREM 1.22 [56]. Let $\mathfrak{B} \rightarrow \mathfrak{B} \rightarrow M$ be a family of vector bundles of rank $r$ over the Riemannian sphere. Then there is an analytic subset $A \subset M$ that is either empty or purely 1-codimensional in each of its points, and a nondecreasing sequence of integers $k_{1}, k_{2}, \cdots, k_{r}$ such that the restriction $\mathfrak{B S}|(M-A) \rightarrow \mathfrak{B}|(M-A) \rightarrow(M-A)$ is locally isomorphic to

$$
U \times \oplus\left\{L_{1}^{k \rho} \mid \rho=1, \cdots, r\right\} \rightarrow U \oplus P^{1} \rightarrow U
$$

where $U$ is an appropriate neighborhood in $M$. In case $r=1, A$ is empty. 
We say that the family $\mathfrak{W} \rightarrow \mathfrak{B} \rightarrow M$ of vector bundles is of type $\left(k_{1}, \cdots, k_{r}\right)$ provided these are the integers corresponding to the local splitting of the family as described in Theorem 1.22.

From Theorem 1.22 one can derive immediately an analogous theorem on families of principal $P G L(r, C)$-bundles over $P^{1}$.

Now consider a family $\mathfrak{F} \rightarrow \mathfrak{B} \rightarrow M$ of principal $G A(1, C)$-bundles over $P^{1}$. It corresponds to an element in $H^{1}\left(\mathfrak{B}, G A(1, C)_{\omega}\right)$ and as before we get a mapping

$$
\pi_{\mathfrak{B}}: H^{1}\left(\mathfrak{B}, G A(1, C)_{\omega}\right) \rightarrow H^{1}\left(\mathfrak{B}, G L(1, C)_{\omega}\right) .
$$

On the other hand, Theorem 1.22 shows that for a polycylinder $U$ and the projection $p: U \times P^{1} \rightarrow P^{1}$ the induced mapping

$$
p^{*}: H^{1}\left(U \times P^{1}, G L(1, C)_{\omega}\right) \rightarrow H^{1}\left(P^{1}, G L(1, C)_{\omega}\right)
$$

is bijective. Using Proposition 1.4 we get therefore a surjective mapping "degree" that is defined as the mapping deg $O p^{*} \circ \pi_{U \times P^{1}}$ of $H^{1}\left(U \times P^{1}, G A(1, C)_{\omega}\right)$ onto $Z$. As in [2] one can show

Proposition 1.23. The set of isomorphy classes of principal $G A(1, C)$-bundles $\mathfrak{F} \rightarrow U \times P^{1} \rightarrow U$ whose degree equals $d$ consists of $p^{-1}\left(L_{1}^{d}\right)$ and the coset space $H^{0}\left(U, \mathfrak{O}_{U}^{\min (0,-d-1)}\right) / H^{0}\left(U, \mathfrak{O}_{U}^{*}\right) .{ }^{5}$

This proposition classifies locally the families of principal $G A(1, C)$ bundles. It can be viewed as the analog of Theorem 1.22 for principal $G A(1, C)$-bundles. From both we can derive to some extent a global classification of families of vector bundles resp. principal $G A(1, C)$ bundles over $P^{1}$, which we shall describe now.

Let us first deal with families $\mathfrak{W} \rightarrow \mathfrak{B} \rightarrow M$ of vector bundles of rank $r$ whose parameter space is a complex manifold. Assuming that the family is of type $\left(k_{1}, \cdots, k_{r}\right)$ the proof of Theorem 1.22 shows that the set of all those points $t$ of the parameter space for which the restriction $W_{t} \rightarrow V_{t}$ of the family is not of type $\left(k_{1}, \cdots, k_{r}\right)$ forms an analytic subset of $M$ that is either purely 1 -codimensional in each of its points or empty. Therefore, families that give rise to different analytic subsets are surely not isomorphic. Furthermore, we see that over $M-A$ the family is locally trivial in the sense of Theorem 1.22. Hence, denoting the group of analytic fiber preserving automorphisms of $L_{1}^{\boldsymbol{k}_{1}} \oplus \cdots \oplus L_{1}^{\boldsymbol{k}_{r} \rightarrow P^{1}}$ that induce the identity mapping of $P^{1}$ by $A\left(k_{1}, \cdots, k_{r}\right)$ and anticipating (see Proposition 2.4) that

\footnotetext{
${ }^{5} \mathfrak{O}_{U}$ shall denote the sheaf of germs of holomorphic functions over $U, \bigcirc_{U}^{k}$ the direct sum of $k$ copies of $\mathfrak{D}_{U}$, and $\mathfrak{D}_{U}^{*}$ the subsheaf of $\mathfrak{D}_{U}$ consisting of invertible germs; $\mathfrak{D}_{U}^{*}$ operates on $\mathfrak{Q}_{U}^{k}$ by left multiplication in the usual way.
} 
$A\left(k_{1}, \cdots, k_{r}\right)$ is a complex Lie group that acts holomorphically on $L_{1}^{k_{1}} \oplus \cdots \oplus L_{1}^{k_{r}} \rightarrow P^{1}$, we observe that the restriction $\mathfrak{B} \mid(M-A)$ $\rightarrow \mathfrak{B} \mid(M-A) \rightarrow(M-A)$ defines in a canonical way an element of $H^{1}\left(M-A, A\left(k_{1}, \cdots, k_{r}\right)_{\omega}\right)$. Obviously, nonisomorphic families give rise to different elements in $H^{1}\left(M-A, A\left(k_{1}, \cdots, k_{r}\right)_{\omega}\right)$ and vice versa. On the other hand, an element $\eta$ in that cohomology set determines a family $\mathfrak{B} \rightarrow \mathfrak{B} \rightarrow(M-A)$ of vector bundles of rank $r$ over $P^{1}$ (whose exceptional set $A$ is empty) and therefore an element $(\eta, \mathfrak{B} \rightarrow M)$ of $H^{1}\left(\mathfrak{B} \mid(M-A), G L(r, C)_{\omega}\right)$. Considering the mapping

$$
H^{1}\left(\mathfrak{B}, G L(r, C)_{\omega}\right) \rightarrow H^{1}\left(\mathfrak{B} \mid(M-A), G L(r, C)_{\omega}\right)
$$

induced by the inclusion $\mathfrak{B} \mid(M-A) \subset \mathfrak{B}$ we see that $(\eta, \mathfrak{B} \rightarrow M)$ arises from a family $\mathfrak{W} \rightarrow \mathfrak{B} \rightarrow M$ if and only if it is hit under the mapping (2). Determining all those elements of $H^{1}\left(\mathfrak{B}, G L(r, C)_{\omega}\right)$ that hit $(\eta, \mathfrak{B} \rightarrow M)$ means to determine all isomorphy classes of families which define the same element $\eta \in H^{1}\left(M-A, A\left(k_{1}, \cdots, k_{r}\right)_{\omega}\right)$. Thus we have

THEOREM 1.24 [56]. The isomorphy classes of families of vector bundles $\mathfrak{B} \rightarrow \mathfrak{B} \rightarrow M$ of type $\left(k_{1}, \cdots, k_{r}\right)$ whose parameter space is a complex manifold are in a bijective relation with the set of triples $(A, \eta, \zeta)$ where $A$ is an analytic subset of $M$ that is either empty or purely 1-codimensional in each of its points, $\eta$ is an element of $H^{1}\left(M-A, A\left(k_{1}, \cdots, k_{r}\right)_{\omega}\right)$, and $\zeta$ is an element of $H^{1}\left(\mathfrak{B}, G L(r, C)_{\omega}\right)$ that hits $(\eta, \mathfrak{B} \rightarrow M)$ under the mapping (2).

However, the question whether a given element of $H^{1}(\mathfrak{B} \mid(M-A)$, $\left.G L(r, C)_{\omega}\right)$ is in the image of (2) seems to be difficult to answer.

Turning to families $\mathfrak{F} \rightarrow \mathfrak{B} \rightarrow M$ of principal $G A(1, C)$-bundles over $P^{1}$ whose parameter space $M$ is a complex manifold we take from Proposition 1.23 that the given family determines in a unique way an element of $H^{0}\left(M, \mathfrak{S}_{M}^{\min (0,-d-1)} / \mathfrak{O}_{M}^{*}\right)$ where $d$ denotes the degree of this family. Families of the same degree that give rise to different sections in $\mathfrak{O}_{M}^{\min (0,-d-1)} / \mathfrak{O}_{M}^{*}$ are not isomorphic. Denoting the group of analytic fiber preserving automorphisms of a principal $G A(1, C)$ bundle of degree $d$ over $P^{1}$, that induce the identity mapping of $P^{1}$, by $G(d)$ and anticipating that $G(d)$ depends only on $d$ and is a complex Lie group that acts holomorphically on the bundle, we find in the same manner as before

THEOREM 1.25. The isomorphy classes of principal GA(1,C)-bundle of degree $d$ over $P^{1}$ whose parameter space $M$ is a complex manifold correspond bijectively to the set

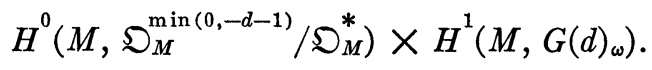


In the general case, however, the best we can hope for is to get a reduction of the structure group of the family. This indeed can be achieved as we see from

THEOREM 1.26 [56]. Let $\mathfrak{F} \rightarrow \mathfrak{B} \rightarrow M$ be a family of fiber bundles over $P^{1}$ whose structure group $G$ is reductive and whose parameter space is either holomorphically complete or a normal projective variety. Then there is a 1-codimensional analytic subset $A$ of $M$ such that the fiber bundle $\mathfrak{F}|(M-A) \rightarrow \mathfrak{B}|(M-A)$ admits a reduction of the structure group $G$ to the normalizer of the Cartan subgroup of $G$.

Moreover, from the results of [16] resp. [56] one can conclude

THEOREM 1.27. Let $\mathfrak{W} \rightarrow \mathfrak{B} \rightarrow M$ be a family of vector bundles of rank $r$ over a family of compact Riemann surfaces whose parameter space $M$ is either holomorphically complete or a normal projective variety. Then there is a 1-codimensional analytic subset $A$ of $M$ such that the restriction $\mathfrak{W}|(M-A) \rightarrow \mathfrak{B}|(M-A)$ admits a reduction of the structure group to the group $\Delta(r, C)$ of $r \times r$ triangular matrices.

2. Groups of fiber preserving automorphisms. The last part of the previous section shows the importance of information concerning the group Aut $(F \rightarrow X)$ of fiber preserving holomorphic automorphisms (in the sense of [62]) of a given bundle $F \rightarrow X$. Denoting the group of holomorphic homeomorphisms of $X$ onto itself by $\operatorname{Aut}(X)$ we get as an easy consequence of Theorem 1.0

TheOREM 2.0. Let $X$ be a noncompact Riemann surface and $F \rightarrow X$ a fiber bundle whose structure group $G$ is connected. Then the group $\operatorname{Aut}(F \rightarrow X)$ is isomorphic to $\operatorname{Aut}(X) \times H^{0}\left(X, G_{\omega}\right)$.

For the rest of this section we assume that the base space $X$ is a compact Riemann surface. First we consider principal bundles $P \rightarrow X$ with structure group $G$. $G$ acts by right translation on $P$; the action of an element $g$ of $G$ shall be denoted by $R_{g} . R_{g}$ induces the differential mapping $R_{b}^{\prime}$ in the (real) tangent bundle of $P$. A tangent field $\mathfrak{X}$ over $P$ is called holomorphic if-with reference to local complex coordinates $z_{1}, \cdots, z_{n}$ in $P$-it can be written as

$$
\sum\left\{\mathfrak{X}^{i}\left(z_{1}, \cdots, z_{n}\right) \frac{\partial}{\partial z_{i}} \mid i=1, \cdots, n\right\}
$$

with suitable holomorphic functions $\mathfrak{X}^{i}\left(z_{1}, \cdots, z_{n}\right)$. The tangent field $\mathfrak{Y}$ over $P$ is called conformal if there is a holomorphic tangent field $\mathfrak{X}$ over $P$ such that $\mathfrak{Y}=\mathfrak{X}+\overline{\mathfrak{X}}$ where $\overline{\mathfrak{X}}$ is the field conjugate to $\mathfrak{X}$. Obviously the set of conformal tangent fields forms a complex Lie algebra. With these notations we get 
Theorem 2.1 [40]. Let $P \rightarrow X$ be a principal G-bundle. Then the group $\operatorname{Aut}(P \rightarrow X)$ equipped with the $\mathrm{C}-\mathrm{O}-$ topology can be given the structure of a complex Lie group that acts holomorphically on P. Its Lie algebra is isomorphic to the Lie algebra of all conformal vector fields $\mathfrak{X}$ over $P$ for which $R_{g}^{\prime} \mathfrak{X}=\mathfrak{X}$ for every $g \in G$.

Denoting the biggest connected subgroup of a topological group $H$ by $H_{0}$ we have moreover

Theorem $2.2[36 ; 42]$. Let $P \rightarrow T^{1}$ be a principal G-bundle. Then Aut $_{0}\left(P \rightarrow T^{1}\right)$ acts transitively provided $P \rightarrow T^{1}$ admits a holomorphic connection.

Under certain circumstances the statement of Theorem 2.2 can be reversed, namely

Proposition $2.3[36 ; 42]$. Let $P \rightarrow T^{1}$ be a principal fiber bundle with structure group a connected abelian group. Then $\operatorname{Aut}_{0}\left(P \rightarrow T^{1}\right)$ acts transitively on the set of fibers of $P \rightarrow T^{1}$ if and only if $P \rightarrow T^{1}$ admits a holomorphic connection. In this case, the group $\operatorname{Aut}_{0}\left(P \rightarrow T^{1}\right)$ is abelian and acts simply transitive on $P$.

Because of Proposition 2.3 it is of interest to describe the subset of $H^{1}\left(T^{1}, A_{\omega}\right), A$ being a connected abelian Lie group, that corresponds to principal bundles admitting holomorphic connections. It can be shown [42] that it is a subgroup of $H^{1}\left(T^{1}, A_{\omega}\right)$ that is isomorphic to $A^{2} / L$ where $L$ is a connected Lie subgroup of $A^{2}$ of complex dimension 1.

In this context it should be remarked that given a vector bundle $W \rightarrow X$ the group Aut $(W \rightarrow X)$ is naturally isomorphic to the group Aut $(P \rightarrow X)$ where $P \rightarrow X$ is the principal $G L(r, C)$-bundle associated with $W \rightarrow X .{ }^{6}$ Moreover, one can show that for a vector bundle $W \rightarrow X$ the group Aut $(W \rightarrow X)$ acts transitively on the set of fibers of $W \rightarrow X$ if and only if, for a Remak decomposition $W=W_{1} \oplus \cdots \oplus W_{k}$ of $W$, each group Aut $\left(W_{\kappa} \rightarrow X\right)$ acts transitively on the set of fibers of $W_{k} \rightarrow X[36]$.

A fiber preserving automorphism of $F \rightarrow X$ determines in a canonical way an element of $\operatorname{Aut}(X)$. That defines a homomorphism $p: \operatorname{Aut}(F \rightarrow X) \rightarrow \operatorname{Aut}(X)$ of transformation groups. Having equipped both Aut $(F \rightarrow X)$ and $\operatorname{Aut}(X)$ with the C-O-topology $p$ obviously induces a homomorphism $\operatorname{Aut}_{0}(F \rightarrow X) \rightarrow \operatorname{Aut}_{0}(X)$.

Proposition 2.4 [40]. Let $P \rightarrow X$ be a principal G-bundle. Then the

- This is, of course, still true in general provided the structure group acts effectively and transitively on the fiber. 
homomorphism $\operatorname{Aut}_{0}(P \rightarrow X) \rightarrow \operatorname{Aut}_{0}(X)$ is surjective if either $X=P^{1}$ and the structure group $G$ is nilpotent or $X=T^{1}$ and $P \rightarrow T^{1}$ admits a holomorphic connection.

In case $X=T^{1}$ the condition given in Proposition 2.4 for $A u t_{0}\left(P \rightarrow T^{1}\right)$ $\rightarrow \operatorname{Aut}_{0}\left(T^{1}\right)$ to be surjective is also sufficient (cf. Proposition 2.3).

In order to determine $\operatorname{Aut}(P \rightarrow X)$ by means of $\operatorname{Aut}(X)$ we have to get results concerning $\operatorname{ker}(\operatorname{Aut}(P \rightarrow X) \rightarrow \operatorname{Aut}(X))$. Here we have

Proposition 2.5 [39]. Let $P \rightarrow X$ be a principal G-bundle. Then $\operatorname{ker}(\operatorname{Aut}(P \rightarrow X) \rightarrow \operatorname{Aut}(X))$ is isomorphic (as a topological group) to the subgroup $\Gamma(P)$ of $H^{0}\left(P, G_{\omega}\right)$ consisting of those mappings $f$ for which

$$
f\left(R_{g} x\right)=g^{-1} \cdot f(x) \cdot g \quad \text { for all } x \in P \text { and } g \in G,
$$

$\Gamma(P)$ being equipped with the C-O-topology.

Proposition 2.6 [39]. Let $P \rightarrow X$ be a principal bundle whose structure group is a simply connected nilpotent Lie group. Then $\operatorname{ker}(\operatorname{Aut}(P \rightarrow X) \rightarrow \operatorname{Aut}(X))$ is connected.

And finally

Proposition 2.7 [40]. The complex dimension of $\operatorname{ker}\left(\operatorname{Aut}_{0}(P \rightarrow X)\right.$ $\left.\rightarrow \operatorname{Aut}_{0}(X)\right)$ equals the complex dimension of $H^{0}(X, \mathfrak{O}(L(P)))$ where $L(P)$ is the vector bundle with fiber the (complex) Lie algebra \&5 of $G$ that is associated to the principal bundle $P \rightarrow X$ by the adjoint representation of $G$ in $\$$.

A means of calculating $\operatorname{Aut}(F \rightarrow X)$ explicitly are classification theorems. For instance, the classification of vector bundles over $P^{1}$ provides us with

Proposition 2.8. Let $W \rightarrow P^{1}$ be a vector bundle of type $\left(k_{1}, \cdots, k_{r}\right)$ and assume that the integers $q_{1}, \cdots, q_{s}$ with $q_{1}+\cdots+q_{s}=r$ are chosen in such a way that $k_{q_{1}+\cdots+q_{\sigma-1}+1}=\cdots=k_{q_{1}+\cdots+q_{\sigma}}$ for $\sigma=1$, . . s. Then $\operatorname{ker}\left(\operatorname{Aut}\left(W \rightarrow P^{1}\right) \rightarrow \operatorname{Aut}\left(P^{1}\right)\right)$ is isomorphic to the group $A\left(k_{1}, \cdots, k_{r}\right)$ of matrices

$$
A(t)=\left(\begin{array}{ccc}
A_{11}(t) & & 0 \\
\vdots & \ddots & \\
A_{s 1}(t) & \cdots & A_{s s}(t)
\end{array}\right), \quad \operatorname{det} A(t) \neq 0
$$

where $A_{i j}(t)$ is a $q_{i} \times q_{j}$-matrix whose entries are polynomials in $t$ of degree $k_{q_{1}+\cdots+q_{i}}-k_{q_{1}+\cdots+q_{j}}$.

$W \rightarrow P^{1}$ being trivial over both $U_{0}$ and $U_{\infty}$ implies that the transition function $g_{0, \infty}(x)$ defining $W \rightarrow P^{1}$ can be chosen as 


$$
\left(\begin{array}{ccc}
x^{k_{1}} & & \\
& \cdot & 0 \\
& 0 & \cdot \\
& & x^{k_{r}}
\end{array}\right) .
$$

Then $A(t)$ maps the element $w$ of the fiber $W_{x}$ into the element $A(x) w$ of $W_{x}$. From this we take that the canonical Lie group structure on $A\left(k_{1}, \cdots, k_{r}\right)$ makes this group act holomorphically on $W \rightarrow P^{1}$.

Corollary. If $k_{1}+\cdots+k_{r} \neq 0$, then Aut $\left(W \rightarrow P^{1}\right)$ is isomorphic (as transformation group) to the set of pairs ( $a, A(t)), a \in G L(1, C)$, $A(t) \in A\left(k_{1}, \cdots, k_{r}\right)$, equipped with the multiplication

$$
(a, A(t)) \cdot\left(a^{\prime}, A^{\prime}(t)\right)=\left(a a^{\prime}, A\left(a^{\prime} t\right) A^{\prime}(t)\right)
$$

and the operation (assuming again that $W \rightarrow P^{1}$ is given by $\left.(3)\right)(a, A(t)) w$ $=A(x) w \in W_{a x}$ for $w \in W_{x}$.

In any case, the image of $\operatorname{Aut}\left(W \rightarrow P^{1}\right) \rightarrow \operatorname{Aut}\left(P^{1}\right)$ contains all transformations $x \rightarrow a x$ where $a \in G L(1, C)$. The last corollary shows that this is precisely the image provided $k_{1}+\cdots+k_{r} \neq 0$. However, if $k_{1}+\cdots+k_{r}=0$ and if $W \rightarrow P^{1}$ is not trivial, then the image may contain in addition the transformations $x \rightarrow a x^{-1}$ where $a \in G L(1, C)$; but these are the only elements in $\operatorname{Aut}\left(P^{1}\right)$ that are hit. The transformations of $\operatorname{Aut}\left(W \rightarrow P^{1}\right)$ that hit the mapping $x \rightarrow x^{-1}$ are exactly the matrices

$$
\tilde{A}(t)=\left(\begin{array}{ccc}
\tilde{A}_{11}(t) & \cdots & \tilde{A}_{1 s}(t) \\
\vdots & & \vdots \\
\tilde{A}_{s 1}(T) & \cdots & \tilde{A}_{s s}(t)
\end{array}\right), \quad \operatorname{det} \tilde{A}(t) \neq 0,
$$

where $\tilde{A}_{i j}(t)$ is a $q_{i} \times q_{j}$-matrix whose entries are polynomials of degree $-k_{q_{1}+\cdots+q_{i}}-k_{q_{1}+\cdots+q_{j}}$. The matrices $\widetilde{A}(t)$ operate on $W \rightarrow P^{1}$ in an analogous way to the matrices $A(t)$.

From these last remarks we see that the mapping $\operatorname{Aut}_{0}(F \rightarrow X)$ $\rightarrow \operatorname{Aut}_{0}(X)$ is in general not surjective.

There is one more case which we should like to mention: principal $G A(1, C)$-bundles over $P^{1}$.

Proposition 2.9. Let $F \rightarrow P^{1}$ be a principal $G A(1, C)$-bundle of degree $d>0$ or $d=-1$. Then the group Aut $\left(F \rightarrow P^{1}\right)$ consists of all pairs $(a, B(t))$ where $a \in G L(1, C)$,

$$
B(t)=\left(\begin{array}{cc}
b & p(t) \\
0 & 1
\end{array}\right),
$$

$b \in G L(1, C)$, and $p(t)$ is a polynomial in $t$ of degree $\leqq d$, equipped with the multiplication 


$$
(a, B(t)) \cdot\left(a^{\prime}, B^{\prime}(t)\right)=\left(a a^{\prime}, B\left(a^{\prime}, t\right) B^{\prime}(t)\right) .
$$

The action of this group can be described as follows: using again the covering $\left\{U_{0}, U_{\infty}\right\}$ of $P^{1}$ and fiber coordinates over these open sets, the element

$$
C=\left(\begin{array}{cc}
\alpha & \beta \\
0 & 1
\end{array}\right)
$$

of the fiber $F_{x}$ is mapped by $(a, B(t))$ into the element $B(x) C$ of the fiber $F_{a x}$.

In case the principal $G A(1, C)$-bundle $F \rightarrow P^{1}$ is of degree zero it is isomorphic to the trivial bundle and hence $\operatorname{Aut}\left(F \rightarrow P^{1}\right)$ isomorphic to $\operatorname{Aut}\left(P^{1}\right) \times G A(1, C)$.

In order to tell the automorphism group of principal $G A(1, C)$ bundles of degree $d \leqq-2$ over $P^{1}$ we remark that, using the covering $\left\{U_{0}, U_{\infty}\right\}$ of $P^{1}$, such a bundle can be given fiber coordinates with respect to which the transition function defining the bundle equals

$$
g_{0, \infty}(x)=\left(\begin{array}{cc}
x^{d} & p_{d}(x) \\
0 & 1
\end{array}\right)
$$

where $p_{d}(x)$ equals

$$
\alpha_{d+1} x^{d+1}+\cdots+\alpha_{-1} x^{-1}
$$

$\left(p_{d}(x)\right.$ is uniquely determined up to a constant factor). Then we have the

Corollary. Suppose that the principal $G A(1, C)$-bundle $F \rightarrow P^{1}$ has degree $d \leqq-2$. Then Aut $\left(F \rightarrow P^{1}\right)$

(i) consists of the identity alone provided $p_{d}(x)$ contains at least two nonvanishing terms,

(ii) consists of all pairs

$$
\left(a^{-k},\left(\begin{array}{ll}
a & 0 \\
0 & 1
\end{array}\right)\right), \quad a \in G L(1, C),
$$

provided $p_{d}(x)=x^{-k}$,

(iii) consists of all pairs

$$
\left(a,\left(\begin{array}{ll}
b & 0 \\
0 & 1
\end{array}\right)\right), \quad a, b \in G L(1, C),
$$

provided $p_{d}(x)$ is identically zero.

The classification of principal $G A(1, C)$-bundles over compact Riemann surfaces of genus $\leqq 2$ as given in [2] allows to determine 
completely the group of fiber preserving automorphisms of these bundles. However, the result is fairly complicated and may therefore be omitted.

Finally we should like to remark that the complex manifolds investigated in [23] correspond bijectively to the bundles described in Proposition 1.6 (for $r=1$ ). The fiber preserving automorphisms of those bundles are determined by Proposition 2.4 and its Corollary. Therefore, these statements also account for the group of fiber preserving automorphisms of bundles with fiber ${ }^{7} P^{n}$ over $P^{1}$ whose structure group is $P G L(n, C)$.

3. Cross-sections in fiber bundles and families of fiber bundles. Theorem 1.0 shows that the fiber bundle $F \rightarrow X$ admits plenty of crosssections provided $X$ is a noncompact Riemann surface and the structure group of $F \rightarrow X$ is connected. Indeed, the set of cross-sections corresponds bijectively to the set of holomorphic mappings of $X$ into the fiber of $F \rightarrow X$. In particular we get

Proposition 3.1. Let $W \rightarrow X$ be a vector bundle of rank $r$ over a noncompact Riemann surface $X$. Then $H^{0}(X, \mathfrak{N}(W))$ is a free module of rank $r$ over the ring $R(X)$ of holomorphic function on $X$.

In addition it is known [14] that in this case $H^{q}(X, \mathfrak{D}(W))=0$ for $q \geqq 1$.

Denoting the sheaf of meromorphic functions on $X$ by $\hat{\mathfrak{D}}$, we define the sheaf $\mathfrak{D}(W)$ to be the sheaf $\hat{\mathfrak{D}} \otimes \mathfrak{O} \mathfrak{D}(W) . \hat{\mathfrak{D}}(W)$ is the sheaf of "meromorphic cross-sections" in $W \rightarrow X$. With this notation we have the

COROLLARY. Let $W \rightarrow X$ be a vector bundle of rank $r$ over a noncompact Riemann surface $X$. Then $H^{0}(X, \hat{\mathfrak{D}}(W))$ is a vector space of dimension $r$ over the field $F(X)$ of meromorphic functions on $X$.

Furthermore, we take from the remark following Proposition 3.1 that $H^{q}(X, \hat{\mathfrak{D}}(W))=0$ for $q \geqq 1$.

Before we turn to compact Riemann surfaces as base spaces we should like to describe briefly a useful device $[17 ; 56]$. Let $p: X \rightarrow Y$ be a (nonconstant) proper $^{8}$ holomorphic mapping and $W \rightarrow X$ a vector bundle. Then there is a vector bundle $p_{*}(W) \rightarrow Y$ such that for every open subset $U$ of $Y$ and $q=0,1, \ldots$ the modules $H^{q}\left(p^{-1}(U), \mathfrak{S}(W)\right)$ and $H^{q}\left(U, \mathfrak{D}\left(p_{*}(W)\right)\right)$ resp. $H^{q}\left(p^{-1}(U), \hat{\mathfrak{D}}(W)\right)$

${ }^{7} P^{n}$ denotes the $n$-dimensional complex projective space.

8 Nonconstant proper holomorphic mappings are exactly the projections of unbounded, but possibly ramified coverings $X \rightarrow Y$ having only finitely many sheets. 
and $H^{q}\left(U, \hat{\mathfrak{D}}\left(p_{*}(W)\right)\right)$ are in a natural bijective correspondence. From this fact and the classification theorem of vector bundles over $P^{1}$ (Theorem 1.3 and Proposition 1.4) one can get a very short proof of the well-known Riemann-Roch formula $[24 ; 65]$

$$
\operatorname{dim}_{C} H^{0}(X, \mathfrak{O}(W))-\operatorname{dim}_{C} H^{1}(X, \mathfrak{O}(W))=\operatorname{deg}(W)+r(1-g)
$$

where $W \rightarrow X$ is a vector bundle of rank $r$ over the compact Riemann surface $X$ of genus $g$.

As far as meromorphic cross-sections in vector bundles are concerned the situation is the same whether the base space is compact or not:

Proposition $3.2[44 ; 48 ; 55]$. Let $W \rightarrow X$ be a vector bundle of rank $r$ over a compact Riemann surface $X$. Then $H^{0}(X, \hat{\mathfrak{D}}(W))$ is a vector space of dimension $r$ over $F(X)$. In particular, given a point $x_{0} \in X$ one can find a basis of $H^{\circ}(X, \hat{\mathfrak{D}}(W))$ whose elements are holomorphic sections over some neighborhood $U$ of $x_{0}$ and which span every fiber $W_{x}$ for $x \in U$.

Again we get in addition that $H^{q}(X, \hat{\mathfrak{N}}(W))=0$ for $q \geqq 1$.

Let $H \rightarrow X$ be the line bundle corresponding to a hyperplane section ( $X$ can be considered as a projective variety). Given a vector bundle $W \rightarrow X$ we denote by $W(n) \rightarrow X$ the vector bundle $W \otimes H^{n} \rightarrow X$. Then one can conclude immediately from Proposition 3.2

Proposition 3.3 [44]. For every vector bundle $W \rightarrow X$ of rank $r$ there is an integer $n$ such that $W(n) \rightarrow X$ is isomorphic to $f^{-1}\left(W_{u}\right)$ where $W_{u} \rightarrow G_{r, N}$ is the universal bundle over the Grassmann variety (of $r$ dimensional vector subspaces of $C^{N}$ ) and $f: X \rightarrow G_{r, N}$ a suitable holomorphic mapping.

A vector bundle $W \rightarrow X$ is called ample if

(i) $H^{q}(X, \mathfrak{S}(W))=0$ for $q \geqq 1$

(ii) for every $x \in X$ the canonical homomorphism $\mathfrak{D}_{x} \otimes_{C} H^{0}(X, \mathfrak{D}(W)$ ) $\rightarrow \mathfrak{D}(W)_{x}$ is surjective. ${ }^{9}$

With this definition we have

Proposition 3.4 [3]. Given a compact Riemann surface $X$ of genus $g$, then for every indecomposable vector bundle $W \rightarrow X$ of rank $r$ and degree $d$ the bundle $W(n) \rightarrow X$ is ample provided $n \geqq(r-1)(3 g-2)-d / r$ $+2 g$.

This leads immediately to the

${ }^{9}$ Given a sheaf $\&$ over $X, \oiint_{x}$ denotes the stalk of $\&$ in $x \in X$. 
CoROllaRY. Let $W \rightarrow X$ be a vector bundle that arises from a homomorphism of $\pi_{1}(X)$ into $G L(r, C)$. Then $W(n) \rightarrow X$ is ample for all $n \geqq(r-1)(3 g-2)+2 g$.

More results of this type can be found in [3].

The statements which we encountered so far assure the existence of plenty of cross-sections. However, it is of interest to obtain some information concerning the dimension of $H^{0}(X, \mathfrak{D}(W))$ in sufficiently general cases. Here we have for instance

Proposition 3.5. Let $W \rightarrow X$ be an indecomposable vector bundle over a compact Riemann surface that arises from a homomorphism of $\pi_{1}(X)$ into $\Delta(r, C), r \geqq 2$. Denoting the dual bundle of $W \rightarrow X$ by $W^{*} \rightarrow X$ we have

and

$$
\operatorname{dim}_{C} H^{0}(X, \mathfrak{D}(W))+\operatorname{dim}_{C} H^{0}\left(X, \mathfrak{D}\left(W^{*}\right)\right) \leqq r
$$

$$
\operatorname{dim}_{C} H^{0}(X, \mathfrak{D}(W)) \geqq 1, \quad \operatorname{dim}_{C} H^{0}\left(X, \mathfrak{D}\left(W^{*}\right)\right) \geqq 1 .
$$

This proposition has been proved in [39] for $X=T^{1}$.

One can show in addition that a vector bundle $W \rightarrow X$ of the type dealt with in Proposition 3.5 has a trivial subbundle of rank $\operatorname{dim}_{C} H^{0}(X, \mathfrak{D}(W))$.

An entirely different type of theorem determining the dimension of cohomology modules are the "vanishing theorems" $[1 ; 11 ; 28 ; 44]$. Via the Riemann-Roch theorem they admit to calculate the dimension of the module of global cross-sections in certain vector bundles. The hypotheses of these theorems are rather complicated wherefore the detailed statements may be omitted.

Turning to different types of fiber bundles we get

Proposition $3.6[2 ; 55]$. Let $F \rightarrow X$ be a fiber bundle with structure group $G A(r, C)$ and fiber $C^{r}$. Then $H^{0}(X, \hat{\mathfrak{D}}(F))$ is an $r$-dimensional affine space over $F(X)$.

By combining Theorem 1.3, Proposition 1.4, and the construction of $p_{*}(W)$, we get

Proposition 3.7. Let $F \rightarrow X$ be a fiber bundle with structure group $P G L(r, C)$ and fiber $P^{r}$. Given any point $x_{0} \in X$ there are $(g+1)(r+1)$, $g$ being the genus of $X$, global cross-sections in $F$ such that for every point $x \neq x_{0}$ of $X$ the values in $x$ of these cross-sections span the fiber $F_{x}$.

Finally we should like to deal with families of fiber bundles. Again we treat first the case where the base family is a family of noncompact Riemann surfaces. Using the notations preceding Proposition 1.20, Theorem 1.21 provides us with 
Theorem 3.8. Let $\mathfrak{B} \rightarrow U_{0}$ be a holomorphic family $\mathfrak{S}$ of complex structures on the noncompact Riemann surface $X_{0}$ whose parameter space is a polycylinder. Let $X$ be a relatively compact and open subset of $X_{0}$ and $\mathfrak{W} \rightarrow \Im\left(U_{0}, X\right) \rightarrow U_{0}$ a family of vector bundles of rank $r$. Then for every $t \in U_{0}$ there is a neighborhood $U$ such that $H^{0}(\mathfrak{S}(U, X), \mathfrak{D}(\mathfrak{W})$ ) is a free module of rank $r$ over the ring $R(\Im(U, X))$ of holomorphic functions on $\Im(U, X)$.

It is clear that a similar statement concerning meromorphic crosssections holds. In addition it may be remarked that under the hypothesis that $U_{0}$ is a trivial family (i.e. $\mathfrak{B}=U_{0} \times X_{0}$ ) the result of Theorem 3.8 is still good for $X=X_{0}$ and $U=U_{0}$.

Turning to base families that are families of compact Riemann surfaces the basic result is

THEOREM 3.9 [30]. Let $\mathfrak{W} \rightarrow \mathfrak{B} \rightarrow U$ be a family of vector bundles over a family of compact Riemann surfaces whose parameter space is a polycylinder. Assume that $k=\operatorname{dim}_{C} H^{q}\left(V_{t}, \mathfrak{D}\left(W_{t}\right)\right)$ is independent of the choice of $t \in U, W_{t} \rightarrow V_{t}$ being the restriction of $\mathfrak{W} \rightarrow \mathfrak{B}$ to the fiber $V_{t}$ corresponding to $t \in U$. Then there are $k$ elements in $H^{q}(\mathfrak{B}, \mathfrak{D}(\mathfrak{W}))$ whose restrictions to $V_{t}$ form a basis of $H^{q}\left(V_{t}, \mathfrak{S}\left(W_{t}\right)\right)$ for every $t \in U$.

In general, however, $\operatorname{dim}_{C} H^{q}\left(V_{t}, \mathfrak{D}\left(W_{t}\right)\right)$ will depend upon $t$. Therefore, it is important to have

THEOREM $3.10[16 ; 56]$. Let $\mathfrak{W} \rightarrow \mathfrak{B} \rightarrow M$ be a family of vector bundles over a family of compact Riemann surfaces whose parameter space is a complex manifold. Then there is a 1-codimensional analytic subset $A$ of $M$ such that for each point $t_{0} \in M$ there is a neighborhood $U$ and elements $s_{1}, \cdots, s_{k}$ in $H^{q}(\mathfrak{B} \mid U, \mathfrak{D}(\mathfrak{W}))$ whose restrictions to $V_{t}$ form a basis of $H^{q}\left(V_{t}, \mathfrak{S}\left(W_{t}\right)\right)$ for every $t$ in $U-A \cap U$.

COROLlARy. If $M$ is either (i) holomorphically complete or (ii) a normal projective variety, then there is a 1-codimensional analytic subset $A$ of $M$ such that

(i) the restriction mapping $H^{q}(\mathfrak{B}, \quad \mathfrak{D}(\mathfrak{W})) \rightarrow H^{q}\left(V_{t}, \mathfrak{D}\left(W_{t}\right)\right)$ is surjective for every $t \in M-A$,

(ii) the restriction mapping $H^{q}(\mathfrak{B}, \hat{\mathfrak{S}}(\mathfrak{W})) \cap H^{q}(\mathfrak{B} \mid M-A, \mathfrak{D}(\mathfrak{W}))$ $\rightarrow H^{q}\left(V_{t}, \mathfrak{D}\left(W_{t}\right)\right)$ is surjective for every $t \in M-A$.

It can be taken from $[2 ; 55]$ and the proof leading to Theorem 3.10 that the statements of Theorem 3.9 and Theorem 3.10 (including the Corollary) are still true for families of bundles whose structure group is $G A(r, C)$ and whose fiber is $C^{r}$. Also there is an analogue of Proposition 3.7 concerning families. 
The last results raise the question how the function $t \rightarrow \operatorname{dim}_{C} H^{q}\left(V_{t}, \mathfrak{D}\left(W_{t}\right)\right)$ behaves for a given family of vector bundles. The first investigations are due to K. KODAIRA and D. C. SPENCER [30] and state the upper semi-continuity of this function. In fact we have

Theorem $3.11[16 ; 37 ; 56]$. Let $\mathfrak{B} \rightarrow \mathfrak{B} \rightarrow M$ be a family of vector bundles over a family of compact Riemann surfaces. Then the set of all points $t$ in $M$ for which $\operatorname{dim}_{C} H^{q}\left(V_{t}, \mathfrak{D}\left(W_{t}\right)\right) \geqq j$ is an analytic subset of $M$.

As far as meromorphic sections in families of vector bundles are concerned the situation is much easier. Here we get immediately from the proof leading to Theorem 1.22 and certain constructions in [56]

Proposition 3.12. Let $\mathfrak{B} \rightarrow \mathfrak{B} \rightarrow{ }^{\omega} U_{0}$ be a family of vector bundles of rank $r$ over a family of compact Riemann surfaces whose parameter space is a polycylinder. Then $H^{0}(\mathfrak{B}, \hat{\mathfrak{D}}(\mathfrak{B}))$ is an $r$-dimensional vector space over $F\left(U_{0}\right)$. In particular there are $r$ meromorphic cross-sections in $\mathfrak{W}$ over $\mathfrak{B}$ that depend holomorphically on $t \in U_{0}$ and form a basis of $H^{0}(\mathfrak{B}, \hat{\mathfrak{N}}(\mathfrak{B}))$.

By "depending holomorphically on $t$ " we mean that the set of (polar) singularities of these sections do not contain a set $\omega^{-1}(t)$; therefore these sections can be restricted to every fiber $V_{t}, t \in U_{0}$, and form then a $F\left(V_{t}\right)$-basis of $H^{0}\left(V_{t}, \hat{\mathfrak{S}}\left(W_{t}\right)\right)$.

Statements similar to Proposition 3.12 hold also for different types of fiber bundles but may be omitted here.

Finally we should like to remark that a fairly complete synopsis of the general differential-geometric methods (currents, harmonic forms) in the theory of vector bundles over Riemann surfaces can be found in [48].

4. Applications. Perhaps the best known application of fiber bundles in the theory of functions is concerned with the theorems of MITTAG-LEFFLER resp. WEIERSTRASS and G. D. BIRKHOFF [8]. There we are given a Riemann surface $X$, a subset $A$ of $X$ that has no point of accumulation in $X$, and for each point $a \in A$ a function $f_{a}$ with values in $C$ resp. $G L(1, C)$ resp. $G L(r, C)$ that is holomorphic in all points of some neighborhood of $a$ except possibly in $a$ itself. The question is then whether there is a holomorphic function $f$ on $X-A$ with values in $C$ resp. $G L(1, C)$ resp. $G L(r, C)$ such that $f-f_{a}$ resp. $f \cdot f_{a}^{-1}$ can be extended into $a$ to a holomorphic function with values in the corresponding group. Moreover we may ask as to whether we can find such a function $f$ that depends holomorphically on certain param- 
eters provided the data $f_{a}$ depend holomorphically on these parameters. Attaching to these data an appropriate principal $G A(r, C)$ bundle and thus covering all three cases Theorem 3.8 and the remark following it we find

Proposition 4.1. Let $X$ be a noncompact Riemann surface, $U$ a polycylinder, $\left\{a_{n}(t)\right\}_{n=1,2}, \ldots$ a family of holomorphic mappings of $U$ into $X$ such that the family of points $\left\{a_{n}(t)\right\}_{n=1,2, \ldots}$ uniformly has no point of accumulation in $X$, and a family $\left\{f_{n}\right\}_{n=1,2, \ldots}$ of holomorphic mappings of $U_{n}-A_{n}$ into $G A(r, C)$ where $U_{n}$ is a neighborhood of the subset $A_{n}=\left\{\left(t, a_{n}(t)\right) \mid t \in U\right\}$ of $U \times X$. Then there is a holomorphic mapping $f$ of

$$
U \times X-U\left\{A_{n} \mid n=1,2, \cdots\right\}
$$

into $G A(r, C)$ such that every $f \cdot f_{n}^{-1}$ can be extended into $A_{n}$ to a holomorphic mapping into $G A(r, C)$.

Here "uniformly having no point of accumulation" means that for every $t_{0} \in U$ there is a neighborhood $U_{0}$ of $t_{0}$ and mutually disjoint open sets $V_{1}, V_{2}, \cdots$ such that every $a_{n}(t)$ remains inside $V_{n}$ as $t$ varies in $U_{0}$. It may be remarked that the hypothesis of uniformly having no point of accumulation can be weakened considerably; in particular, for some $t$ the $a_{n}(t)$ do not have to be mutually different.

If $X$ is a compact Riemann surface we cannot expect the same result as in the case of a noncompact Riemann surface. Yet we have an analogue of Proposition 4.1. For that purpose we observe that it makes perfect sense to talk about meromorphic functions with values in $G A(r, C)$ : interpreting $G A(r, C)$ as a subset of $C^{r(r+1)}$ we mean by a meromorphic function with values in $G A(r, C)$ an $r(r+1)$ tuple of meromorphic functions on the complex space under consideration which takes on values in $G A(r, C)$ except for an analytic subset of positive codimension. Then we have

Proposition 4.2. Hypotheses as in Proposition 4.1, except that $X$ is now a compact Riemann surface. Then there is a meromorphic mapping $f$ of

$$
U \times X-U\left\{A_{n} \mid n=1,2, \cdots\right\}
$$

into $G A(r, C)$ having the properties

(i) there is a finite subset $X_{0}$ of $X$ such that f restricted to $U \times\left(X-X_{0}\right)$ is a holomorphic mapping into $G A(r, C)$,

(ii) every $f \cdot f_{n}^{-1}$ can be extended into $A_{n}-A_{n} \cap\left(U \times X_{0}\right)$ to a holomorphic mapping into $G A(r, C)$.

Proposition 4.2 is of interest only in the case where the functions $f_{n}$ have essential singularities on $A_{n}$. 
One more quite well-known application $[13 ; 48 ; 54]$ is the following. An abelian integral (resp. multiplicative function) on a Riemann surface $X$ defines in a canonical way a homomorphism of $\pi_{1}(X)$ into $C$ (resp. $G L(1, C)$ ) and therefore a fiber bundle with fiber $C$ (see also 1). Abelian integrals (multiplicative functions) with the given periods correspond bijectively to cross-sections in that fiber bundle. More generally we could consider mappings $\phi: U \times \pi_{1}(X) \rightarrow C$ (resp. $G L(1, C)), U$ being a polycylinder, which have the property that for fixed $t \in U$ the mapping $\alpha \rightarrow \phi(t, \alpha)$ is a homomorphism and for fixed $\alpha \in \pi_{1}(X)$ the mapping $t \rightarrow \phi(t, \alpha)$ is a holomorphic mapping; in this case we say that the additive (multiplicative) periods depend holomorphically upon $t \in U$. Again the problem is to find holomorphic functions on $U \times \tilde{X}, \tilde{X}$ being the universal covering of $X$, that considered as abelian integrals (multiplicative functions) on $X$ have the prescribed periods for every $t \in U$. The answers to these questions are exactly the same ones as those given in Proposition 4.1 and Proposition 4.2. In particular the analogue to Proposition 4.1 extends a wellknown result [6] concerning integrals of the first kind on noncompact Riemann surfaces. If we are interested in integrals of the first kind (holomorphic multiplicative functions) on compact Riemann surfaces, Theorem 3.9 implies as an easy consequence

Proposition 4.3. Let $\mathfrak{B} \rightarrow U$ be a family of compact Riemann surfaces whose base space $U$ is a polycylinder. Suppose that the homomorphism $\phi: U \times \pi_{1}\left(V_{t_{0}}\right) \rightarrow C$ (resp. $\left.G L(1, C)\right)$ depends holomorphically on $t \in U$. Suppose furthermore that for every $t \in U$ there is an abelian integral of the first kind (a holomorphic multiplicative function) on $V_{t}$ having the periods prescribed by $\alpha \rightarrow \phi(t, \alpha)$. Then there is a holomorphic function on the universal covering $\tilde{\mathfrak{B}}$ of $\mathfrak{B}$ that considered as an integral of the first kind (holomorphic multiplicative functions) on each fiber $V_{t}$ has the periods prescribed by $\phi$.

It is obvious that the last type of question can be posed and treated in exactly the same way as before if we replace the homomorphism $\varphi: \pi_{1}(X) \rightarrow C$ by a homomorphism of $\pi_{1}(X)$ into an arbitrary Lie group $G$. Then we would end up with automorphic functions on $X$ with values in some space $Y$ on which $G$ operates as a group of holomorphic automorphisms.

A problem related to the last one is the following. Consider a system of $r$ linear homogeneous differential equations on $X$, i.e. a system

$$
d y_{j}=\sum\left\{y_{k} \omega_{j k} \mid k=1, \cdots, r\right\}, \quad j=1, \cdots ; r
$$

where the $\omega_{j k}$ 's are meromorphic differential forms on $X$. As is well 
known there is always a fundamental system, that is, there are $n$ solutions of (4) of which every other solution is a linear combination over the field of complex numbers. Denoting by $A$ the set of poles of the coefficients $\omega_{j k}$, all solutions are holomorphic functions on the universal covering of $X-A$. Therefore a fundamental system of (4) determines a homomorphism of $\pi_{1}(X-A)$ into $G L(r, C)$ whose class (modulo inner automorphisms) is independent of the choice of the fundamental system; this class is usually called the monodromy. In the points of $A$ we have to expect singularities of the solutions. In case the singularities of all solutions are "pole-like," ${ }^{10}(4)$ is said to be a FUCHSian system. The problem is to find out whether for a given subset $A$ of $X$ having no points of accumulation in $X$ and a given homomorphism of $\pi_{1}(X-A)$ into $G L(r, C)$ there is a FUCHsian system on $X$ whose singularities are in $A$ and whose monodromy is the prescribed one. The question has been answered affirmatively in case $X=P^{1}$ by classical means $[9 ; 22 ; 50 ; 52]$. Moreover the question arises in which way the FUCHSian system depends upon the points of $A$ and the monodromy [34]. By constructing a family of vector bundles belonging to the given monodromy and extending it properly in to the set of singularities we find

TheOREM $4.4[48 ; 54]$. Let $X$ be a noncompact Riemann surface, $U$ a polycylinder, $\left\{a_{n}(t)\right\}_{n=1,2,} \ldots$ a family of holomorphic mappings of $U$ into $X$ such that the family of points $\left\{a_{n}(t)\right\}_{n=1,2, \ldots}$ uniformly has no point of accumulation, and $\mu: U \times \pi_{1}\left(X-A_{t_{0}}\right) \rightarrow G L(r, C)$ a homomorphism that depends holomor phically on twhere $A_{t}=\mathrm{U}\left\{a_{n}(t) \mid n=1,2, \cdots\right\}$. Then there is a system (4) whose coefficients depend holomorphically on $t \in U$ such that for every fixed $t \in U$ the corresponding system is FUCHsian, has singularities only in the set $A_{t}$, and gives rise to the monodromy prescribed by the homomorphism $\alpha \rightarrow \mu(t, \alpha) .{ }^{11}$ In particular the poles of the coefficients of the system have order 1.

Theorem $4.5[48,54]$. Same hypotheses as in Theorem 4.4, except that $X$ is now a compact Riemann surface. Then there is a system (4) whose coefficients depend holomorphically on $t \in U$ such that for every fixed $t \in U$ the corresponding system is FUCHsian and gives rise to the monodromy prescribed by the homomorphism $\alpha \rightarrow \mu(t, \alpha)$.

${ }^{10}$ A (not necessarily single valued) function $f(z)$ is said to have a pole-like singularity at $z=0$ if for some integer $k$ the function $z^{k} f(z)$ tends uniformly to zero as $z$ tends to zero with uniformly bounded argument.

${ }^{11}$ Note that under the hypotheses of our theorem $\pi_{1}\left(X-A_{t}\right)$ and $\pi_{1}\left(X-A_{t_{0}}\right)$ are canonically isomorphic. 
Similar results can be gotten by the same method if we prescribe essential singularities instead of pole-like singularities that lead to Fuchsian type. At the same time we can replace $X$ by a family of compact Riemann surfaces. Also the hypothesis of uniformly having no point of accumulation can be weakened. In particular, under certain additional hypotheses one can also treat cases in which the points $a_{n}(t)$ are not mutually distinct for every choice of $t$. This is of interest in dealing with TEICHMÜLLER's [63] Windungsstückkoordinaten-approach to the problem of moduli of Riemann surfaces.

Assuming that $p: X \rightarrow Y$ is a nonconstant proper holomorphic mapping of connected Riemann surfaces the construction of $p_{*}(1)^{12}$ may be viewed as a special case of the extension of vector bundles needed for the proof of Theorem 4.4 and Theorem 4.5. The mapping $p$ induces an injective homomorphism $p^{*}: R(Y) \rightarrow R(X)$ of rings of holomorphic functions (which of course is only of interest in case $Y$ is not compact). By means of Proposition 3.1 we get

Proposition 4.6 [57]. Let $Y$ be a noncompact Riemann surface. Then a ring extension $R$ of $R(Y)$ is $R(Y)$-isomorphic ${ }^{13}$ to a ring extension $R(X) \supset R(Y)$ defined by an unbounded (but possibly ramified) covering $X \rightarrow Y$ having $n$ sheets if and only if

(i) $R$ has no zero divisors,

(ii) $R$ is integrally closed,

(iii) (in its structure as an $R(Y)$-module) $R$ is a free $R(Y)$-module of rank $n$.

If $R$ satisfies these conditions, then $X \rightarrow Y$ is uniquely determined up to a fiber preserving isomorphism $(X \rightarrow Y$ being considered as a singular fibering).

In some sense Proposition 4.6 gives a criterion as to whether a Riemann surface $X$ can be realized as an unbounded covering of a given noncompact Riemann surface $Y$. The corresponding question for compact Riemann surfaces $Y$ has been answered a long time ago. In the latter case such a realization is always possible if $Y=P^{1}$ and $X$ is any compact Riemann surface. It is trivial that $X$ which is assumed to have genus $g$ can be realized as a covering of $P^{1}$ having at most $g+1$ sheets. However, it can be shown $[12 ; 21]$ that "in general" such a realization having at most $[(g+3) / 2]$ sheets $^{14}$ can be found. Using

\footnotetext{
121 denotes the trivial line bundle over a Riemann surface.

${ }^{13} R(Y)$-isomorphic shall mean that the isomorphism leaves $R(Y)$ element-wise fixed.

${ }^{14}[\lambda]$ denotes the biggest integer $\leqq \lambda$.
} 
certain methods in complex function theory of several variables Theorem 3.11 leads to

TheOREM 4.7 [37]. Let $\mathfrak{B} \rightarrow M$ be a family of compact Riemann surfaces of genus $g$. Then the set of all points $t \in M$ for which $V_{t}$ admits a realization over $P^{1}$ that has at most $d$ sheets forms an analytic subset $B_{d}(\mathfrak{B} \rightarrow M)$ of $M$. In particular, if $\mathfrak{B}_{g} \rightarrow M_{g}$ is the Teichmiiller family for genus $g$, then $B_{[(g+3) / 2]}\left(\mathfrak{B}_{g} \rightarrow M_{g}\right)=M_{g}$. Furthermore there is an analytic subset $A_{g}$ of $M_{g}$ such that for every $t \in M_{g}-A_{g}$ the corresponding Riemann surface can be realized as a covering of $P^{1}$ that has exactly $[(g+3) / 2]$ sheets.

The ideas of [37] also lead to results if we consider an arbitrary compact Riemann surface $Y$ instead of $P^{1}$.

A result that is closely related to Theorem 4.7 and can also be gotten from Theorem 3.11 is

TheOREM $4.8[51 ; 56]$. Let $\mathfrak{B} \rightarrow M$ be a family of compact Riemann surfaces. Then the set of all points in $\mathfrak{B}$ that are Weierstrass points of the fiber to which they belong form an analytic subset of $\mathfrak{B}$.

Similar statements can be proved as we partly prescribe the gaps for the Weierstrass points.

There is one more application of fiber bundle techniques to the theory of systems of linear differential equations. Let (4) be a system that is defined in the annulus $0<|z|<r$; we write it in the form

$$
d \mathfrak{y}=\Omega(z) \mathfrak{y}
$$

where $\Omega(z)$ is now an $n \times n$ matrix of meromorphic differential forms in $0<|z|<r$. Then $\left(4^{\prime}\right)$ is called equivalent to the system with matrix $\tilde{\Omega}(z)$ if there is a holomorphic and holomorphically invertible matrix $H(z)$ defined in a full neighborhood of $z=0$ such that

$$
\tilde{\Omega}(z)=H^{-1}(z) \Omega(z) H(z)-H^{-1}(z) d H(z)
$$

holds. The problem is to find normal forms of systems $\left(4^{\prime}\right)$ under this equivalence relation. This question has been dealt with in various papers (see $[10 ; 41]$ ). Now let us turn to the following situation. Suppose we are given a holomorphic mapping $\tilde{a}(t)$ of a polycylinder $U$ into $C^{1}$. Let $V$ be a neighborhood of the subset $\tilde{A}=\{(t, \tilde{a}(t) \mid t \in U\}$ of $U \times C^{1}$ and $\Omega(t, z)$ an $n \times n$ matrix of meromorphic differential forms along $C^{1}$ that are defined ${ }^{15}$ in $V-\tilde{A}$ and depend holomorphically on $t \in U$. For every $t \in U$ we have a power series expansion

${ }^{15}$ A meromorphic differential form along $C^{1}$ is a differential form $f(t, z) d z$ where $f(t, z)$ is a meromorphic function. 


$$
\Omega(t, z)=\sum\left\{\Omega_{\nu}(t) \cdot(z-\tilde{a}(t))^{\nu} d z \mid \nu=q(t), \cdots\right\}
$$

where $q(t)$ is either $-\infty$ or the uniquely determined integer for which $\Omega_{q(t)}(t)$ is not the zero matrix. Using these notations we get by employing a suitable vector bundle, Theorem 1.22, and the method used in $[10]$

TheOREM 4.9. Suppose that $q(t) \geqq q$ for all $t \in U$. Then there is an integer $N$, an at most 1-codimensional analytic subset $A$ of $U$, and a meromorphic nonsingular matrix $H(t, z)$ defined in some neighborhood of $\tilde{A}$ such that

$$
\tilde{\Omega}(t, z)=H^{-1}(t, z) \Omega(t, z) H(t, z) H(t, z)-H^{-1}(t, z) d H(t, z)
$$

can be written as

$$
\sum\left\{\widetilde{\Omega}_{\nu}(t) \cdot(z-\tilde{a}(t))^{\nu} d z \mid \nu=\min (0, q), \cdots, N\right\}
$$

where the matrices $\widetilde{\Omega}_{\nu}(t)$ are meromorphic in $U$. Moreover for every fixed $t \in U-A$ the matrix $H(t, z)$ establishes an equivalence between $\Omega(t, z)$ and $\widetilde{\Omega}(t, z)$.

The meaning of Theorem 4.9 is of course that we are able to get normal forms "uniformly" except for lower dimensional subsets of the parameter space. In particular the pole orders in $\infty$ of the transformed system is uniformly bounded (by $N$ ). It should be remarked that for points $t \in A$ the matrix $H(t, z)$ in general is neither holomorphic nor invertible. However, conditions can be given which assure that the exceptional set $A$ is empty.

The classical approach to the problem treated in Theorem 4.4 and Theorem 4.5 makes use of what is usually called the Hilbert boundary value problem. It can be described as follows. Let $C_{1}, \cdots, C_{n}$ be mutually disjoint Lyapunov curves in the Riemannian sphere $P^{1}$. Then $P^{1}-\bigcup\left\{C_{\nu} \mid \nu=1, \cdots, n\right\}$ consists of $n+1$ connected components $D_{0}, \cdots, D_{n}$ which may be arranged in such a way that the common border of $D_{0}$ and $D_{\nu}$ is $C_{\nu}, \nu=1, \cdots, n$. Given continuous mappings

$$
B_{\nu}: C_{\nu} \rightarrow G A(r, C),
$$

is it possible to find holomorphic mappings $f_{\nu}: D_{\nu} \rightarrow C^{r}$ that can be extended to continuous mappings of the closure $\bar{D}_{\nu}$ of $D_{\nu}$ and satisfy the conditions

$$
f_{\nu}\left(s_{\nu}\right)=B_{\nu}\left(s_{\nu}\right) f_{0}\left(s_{\nu}\right) \quad \text { for every } s_{\nu} \in C_{\nu} \text { and } \nu=1, \cdots, n ?
$$

An affirmative answer to this question has been given provided the mappings $B_{\nu}$ are Hölder-continuous (this hypothesis can be weakened) $[35 ; 43 ; 61]$. The same question can be posed assuming that we are 
given an arbitrary Riemann surface $X$, a locally mutually disjoint family $\left\{C_{n}\right\}_{n=1,2, \ldots}$ of curves on $X$ (i.e. every point $x$ in $X$ has a neighborhood that is hit by at most one curve of the family) each of which is a closed subset of $X$ that separates $X$ locally, and continuous mappings $B_{n}: C_{n} \rightarrow G A(r, C)$ that depend holomorphically upon a parameter $t$ ranging in a polycylinder $U ;{ }^{16}$ it should be remarked that we do not require that the curves $C_{n}$ separate $X$ globally. With these data we associate [58] a family of $G A(r, C)$-bundles over $U \times X$, the fiber of which is $C^{r}$, in such a way that the set of those solutions of the problem which depend holomorphically on $t \in U$ correspond bijectively to the set of cross-sections in this family. In fact, the sheaf of germs of cross-sections in this family of bundles is nothing but the sheaf of germs of local solutions of our problem. Then our previous theorems concerning cross-sections in families of fiber bundles provide us with

TheOREM 4.10 [58]. Let $X$ be a noncompact Riemann surface, $\left\{C_{n}\right\}_{n=1,2, \ldots}$ a family of curves on $X$ subject to the conditions stated previously, and $B_{n}: U \times C_{n} \rightarrow G A(r, C), n=1,2, \cdots$ Hölder-continuous mappings that depend holomorphically upon $t \in U$. Then the set of holomorphic mappings $f$ of $U \times\left(X-\cup\left\{C_{n} \mid n=1,2, \cdots\right\}\right)$ into $C^{r}$, fulfilling for every $n=1,2, \cdots$

(i) given two (mutually disjoint) one-sided neighborhoods $V_{n}^{-}$and $V_{n}^{+}$of $C_{n} f(t, x)$ can be extended to continuous mappings $f^{-}(t, x)$ resp. $f^{+}(t, x)$ of $\mathfrak{u} \times \bar{V}_{n}^{-}$resp. $u \times \bar{V}_{n}^{+}$into $G A(r, C)$ that depend holomorphically on $t \in U$

(ii) for every point $x \in C_{n}$

$$
f^{+}(t, x)=B_{n}(t, x) f^{-}(t, x),
$$

forms a free affine space ${ }^{17}$ of rank $r$ over the ring $R(U \times X)$ of holomorphic functions on $U \times X$. In case every mapping $B_{n}$ takes on values only in $G L(r, C)$ this affine space is a free module of rank $r$ over $R(U \times X)$.

A similar statement can be proved for compact Riemann surfaces $X$ if we admit mermorphic solutions of our problem. If we want to deal with holomorphic solutions in the case of compact Riemann surfaces we will meet certain cohomology conditions that are necessary and sufficient for the existence of such solutions. If $X=P^{1}$ and $r=1$ the cohomology class responsible for the existence of solutions is

16 This shall mean that for every fixed $s_{n}$ the mapping $t \rightarrow B_{n}\left(t, s_{n}\right)$ is holomorphic.

${ }_{17} \mathrm{~A}$ free affine space $W$ over a ring $R$ is an affine space for which there are $r+1$ elements $w_{0}, \cdots, w_{r}$ such that every element $w \in W$ can be written in exactly one way as $a_{0} w_{0}+\cdots+a_{r} w_{r}$ with $a_{\rho} \in R, \rho=1, \cdots, r$, and $a_{0}+\cdots+a_{r}=1$. 
known as the index [43]. It may be remarked that the method indicated here also leads to results in dealing with families $\left\{C_{n}\right\}_{n=1,2}, \ldots$ of curves that depend holomorphically on a parameter ranging in a polycylinder. At the same time one can subject the Riemann surface $X$ to deformations of the complex structure.

The theory of systems of linear differential equations is also linked with the theory of connections. Suppose that the vector bundle $W \rightarrow X$ is defined by the transition functions $g_{i j}(x)$. Then a holomorphic connection in $W \rightarrow X$ is given by assigning to each element $U_{i}$ of the covering a matrix $c_{i}$ of holomorphic differential forms (of degree 1) in $U_{i}$ such that

$$
g_{i j}(x) c_{j}(x)-c_{i}(x) g_{i j}(x)=d g_{i j}(x)
$$

holds in $U_{i} \cap U_{j}$. We speak of a meromorphic connection if we assign to each $U_{i}$ a matrix of meromorphic differential forms (of degree 1) in in $U_{i}$ such that (6) holds. It can be seen easily that every vector bundle over a Riemann surface admits meromorphic connections. For a vector bundle $W \rightarrow X$ over a compact Riemann surface and a meromorphic connection in $W \rightarrow X$ one can develop a theory that is very similar to and in fact a generalization of the classical [21] theory of algebraic functions and their generalizations $[31 ; 53]$ (the classical theory of algebraic functions is gotten by realizing a given compact Riemann surface $Y$ as covering $p: Y \rightarrow P^{1}$ and choosing a suitable connection in the vector bundle $\left.p_{*}(1) \rightarrow P^{1}\right)$. On the other hand if we are given a linear system of differential equations on a compact Riemann surface $X$ we can associate with it a vector bundle in such a way that the solutions of the system correspond bijectively to the global cross-sections; the differentiation then gives rise to a meromorphic connection in this bundle. This set-up is of some interest for what is usually called "special functions" because they are solutions of systems of linear differential equations. And again one can study connections of families of vector bundles that depend holomorphically on parameters, thus incorporating statements concerning special functions on their dependence on certain parameters.

Another problem in theory of functions that leads to connections is the investigation of "nomographic structures" on a Riemann surface [64]. This is essentially the study of the invariants of certain systems of linear differential equations.

As it can be shown [59] fiber bundles play quite a role in the investigation of certain functional equations. We choose as a typical example difference equations on Riemann surfaces. For that purpose let $D$ be a connected open subset of $C^{1}$ that is invariant under the 
translation $z \rightarrow z+1$. Furthermore let $p: X \rightarrow D$ be an unbounded but possibly ramified covering of $D$ and $T: X \rightarrow X$ a holomorphic mapping such that $p(T(x))-p(x)=1$. Then we consider the equation

$$
f(T(x))=\phi(x, f(x))
$$

where $\phi$ is a holomorphic mapping of $X \times Y$ into $Y, Y$ being a complex space. (7) is called a difference equation with values in $Y$. A solution of (7) shall be a holomorphic mapping $f$ of $X$ into $Y$ such that (7) is satisfied for every $x \in X$. With (7) one can associate [59] a fibration ${ }^{18}$ $F_{\phi} \rightarrow X / T$ whose base space $X / T$ is gotten from $X$ by identifying two points $x$ and $x^{\prime}$ provided there is a non-negative integer $n$ such that $x=T^{n}\left(x^{\prime}\right)$. The fibration $F_{\phi} \rightarrow X / T$ has the property that cross-sections in it and solutions of (7) are in a natural bijective correspondence. In a similar way one can deal with the case in which the function $\phi=\phi(t, x, y)$ in (7) depends holomorphically upon a parameter $t$ ranging over a polycylinder $U$ (i.e. $\phi$ is a holomorphic mapping of $U \times X \times Y$ into $Y$ ). Then we ask for holomorphic mappings of $U \times X$ into $Y$ fulfilling (7). In this case the base space of the fibration associated with ( 7$)$ is $U \times(X / T)$. Obviously $U \times(X / T)$ is holomorphically complete and has vanishing integral homology from dimension 2 on. Next we remark that the fibration $F_{\phi} \rightarrow U \times(X / T)$ is actually a fiber bundle provided $(t, x) \rightarrow \phi(t, x, \cdot)$ is a holomorphic mapping of $U \times X$ into a complex Lie group $G$ that acts holomorphically on $Y$. Therefore, by applying the fact that $F_{\phi} \rightarrow U \times(X / T)$ is trivial under these circumstances we get

THEOREM 4.11 [59]. Let $G$ be a connected Lie group that acts holomorphically upon the complex space $Y$, let $U$ be a polycylinder, and $X$ a noncompact Riemann surface together with a holomorphic mapping $T: X \rightarrow X$ subject to the conditions described above. Let furthermore $\phi: U \times X \rightarrow G$ be a holomorphic mapping. ${ }^{19}$ Given a point $x_{0} \in X$ and $a$ holomorphic mapping $f_{0}: U \rightarrow Y$ there is a solution $f$ of (7) fulfilling $f\left(t, x_{0}\right)=f_{0}(t)$.

COROllary. Let $U$ be a polycylinder and $X$ a noncompact Riemann surface together with a holomorphic mapping $T: X \rightarrow X$ subject to the conditions described above. Let furthermore $A: U \times X \rightarrow G L(r, C)$ be a holomorphic mapping. Then the family of systems of linear difference equations

$$
f_{j}(T(x))=\sum\left\{a_{j k}(t, x) f_{k}(t, x) \mid k=1, \cdots, r\right\}, \quad j=1, \cdots, r,
$$

${ }^{18}$ A fibration is a surjective holomorphic mapping $F \rightarrow M$ that is a fibration in the usual topological sense.

${ }^{19}$ The image of $y$ under $\phi(t, x)$ is here denoted by $\phi(t, x, y)$. 
where $\left(\left(a_{j k}(t, x)\right)\right)_{j, k=1, \cdots, r} A(t, x)$, has a fundamental system of solutions that depends holomorphically on $t \in U$.

By a fundamental system we mean $r$ solutions $\left(f_{\rho_{1}}(x), \cdots, f_{\rho_{r}}(x)\right)$, $\rho=1, \cdots, r$, for which the determinant $\operatorname{det}\left(f_{\rho \sigma}(x)\right)_{\rho \sigma=1, \cdots, r}$ has no zeros in $X$. This corollary has been proved in special cases in $[25 ; 38$; 49]. Moreover it should be remarked that similar results can be obtained in case the span of (7), i.e. $p(T(x))-p(x)$, depends on $t \in U$.

As we have seen in the corollary to Theorem 4.11 there are fundamental systems. From the very definition we see that given two fundamental systems $\mathfrak{f}_{1}$ and $\mathfrak{f}_{2}$ the matrix $C(t, x)=\mathfrak{f}_{1}^{-1}(t, x) \mathfrak{f}_{2}(t, x)$ is holomorphic and holomorphically invertible and fulfills

$$
C(t, T(x))=C(t, x) ;
$$

it is obvious that every holomorphic and holomorphically invertible matrix $C(t, x)$ fulfilling (9) can be gotten in this way. The Riemann surface $X$, being realized by $p: X \rightarrow D$ as a covering of $D$, gives rise to the group $\mathfrak{D}$ of covering transformations (i.e. holomorphic automorphisms $\psi: X \rightarrow X$ fulfilling $p \circ \psi=p)$. We assume that the matrix of coefficients $A(t, x)$ of $(8)$ is of the form $\tilde{A}(t, p(x))$ where $\tilde{A}$ is a holomorphic mapping of $U \times D$ into $G L(r, C)$. Choosing a fundamental system $\mathfrak{f}(t, x)$ of $(8)$, for every $\psi \in \mathfrak{D}$ the matrix $\mathfrak{f}\left(\psi^{-1}(x)\right)$ is again a fundamental system. Hence $C(t, x)=\mathfrak{f}^{-1}(t, x) \mathfrak{f}\left(t, \psi^{-1}(x)\right)$ satisfies (9). Obviously

$$
C_{\psi_{1} \psi_{2}}(t, x)=C_{\psi_{1}}(t, x) C_{\psi_{2}}\left(t, \psi_{1}^{-1}(x)\right)
$$

holds. Here we have a question that is very similar to the one answered in Theorem 4.4 and Theorem 4.5, namely: to which extent is it possible to prescribe the "monodromy" $C_{\psi}(t, x)$ ? Again, by constructing appropriate fiber bundles and using the results of $\S 3$ we find

TheOREM 4.12 [59]. Let $X, U, \mathfrak{D}$, be as described above. Given any mapping $C$ of $\mathfrak{D} \times U \times X$ into $G L(r, C)$ that depends holomorphically on $(t, x) \in U \times X$ and fulfills (9) and (10), there is a system of linear difference equations on $X$ depending holomorphically on $t \in U$ that has a fundamental system which depends holomorphically upon $t \in U$ and gives rise to the prescribed monodromy $C$.

There is another possibility for systems of difference equations. Let $\omega_{1}$ and $\omega_{2}$ be two nonzero complex numbers whose ratio is not real. Suppose that $D$ is a connected open subset of $C^{1}$ that is mapped into itself by both transformations, $z \rightarrow z+\omega_{1}$ and $z \rightarrow z+\omega_{2}$. Now we are interested in unbounded coverings $p: X \rightarrow D$ on which we have two holomorphic mappings $T_{1}: X \rightarrow X$ and $T_{2}: X \rightarrow X$ such that $p\left(T_{1}(x)\right)$ 
$-p(x)=\omega_{1}$ and $p\left(T_{2}(x)\right)-p(x)=\omega_{2}$ hold. We consider then the system of equations

$$
f\left(T_{1}(x)\right)=\phi_{1}(x, f(x)), \quad f\left(T_{2}(x)\right)=\phi_{2}(x, f(x))
$$

where the functions $\phi_{1}$ and $\phi_{2}$ are subject to

$$
\phi_{1}\left(T_{2}(x), \phi_{2}(x, y)\right)=\phi_{2}\left(T_{1}(x), \phi_{1}(x, y)\right) .
$$

In this situation too we can apply the previously mentioned methods, ask the corresponding questions, and get the analogous answers [59].

In some sense difference equations are closely related to a concept about which we want to speak now. Let $Y$ be a Riemann surface and $H$ a properly discontinuous group of holomorphic automorphisms of $Y$ (i.e. given any two points $y_{1}, y_{2}$ in $Y$ they have neighborhoods $V_{1}$ resp. $V_{2}$ such that $V_{1} \cap \phi\left(V_{2}\right) \neq \varnothing$ holds only for finitely many $\left.\phi \in H\right)$. Then it is well known that the quotient space $X=Y / H$ in a canonical way can be given the structure of a Riemann surface such that the quotient mapping $q: Y \rightarrow X$ is a holomorphic mapping. Let now $G$ be a complex Lie group. Then a factor of automorphy for $(Y, H)$ with values in $G$ is a mapping $k: Y \times H \rightarrow G$ that depends holomorphically upon $y \in Y$ and fulfills

$$
k\left(y, \phi_{1} \phi_{2}\right)=k\left(y, \phi_{2}\right) k\left(\phi_{2}(y), \phi_{1}\right) .
$$

Two such factors $k_{1}$ and $k_{2}$ are called equivalent if there is a holomorphic mapping $l: Y \rightarrow G$ such that

$$
k_{1}(y, \phi)=l^{-1}(y) k_{2}(y, \phi) l(\phi(y))
$$

holds. Then one can prove

Proposition 4.13 [32]. The set of equivalence classes of factors of automorphy for $(Y, H)$ with values in $G$ is in a natural bijective relation with the set of isomorphy classes of those principal G-bundles $F$ over $X$ for which the induced bundle $q^{-1}(F)$ is trivial.

More results in this direction can be found in [33].

Proposition 4.13 shows that it is reasonable to restrict ourselves to the case where $X$ is a compact Riemann surface. As far as applications are concerned the most interesting case is $G=G L(r, C)$. Here we call a factor of automorphy reducible if it is equivalent to a factor of the form

and get

$$
\left(\begin{array}{cc}
A_{\phi}(y) & B_{\phi}(y) \\
0 & C_{\phi}(y)
\end{array}\right)
$$


TheOREM $4.14[19 ; 20]$. Let $Y$ be a simply connected Riemann surface and $Y / H$ be compact. Then every irreducible factor of automorphy for $(Y, H)$ with values in $G L(r, C)$ is equivalent to one of the form $C(\phi) \cdot k(y, \phi)$ where $C: H \rightarrow G L(r, C)$ is an antihomomorphism and $k(y, \phi)$ a factor of automorphy for $(Y, H)$ with values in $G L(1, C)$.

The classification of the latter ones, however, is classical: in case $Y=C^{1}$ the factors of automorphy for $(Y, H)$ with values in $G L(1, C)$ are of the form

$$
k(z, \phi)=\exp (a(\phi)+b(\phi) z+h(\phi(z))-h(z))
$$

where $h(z)$ is an entire function and $a(\phi)$ and $b(\phi)$ are suitable complex numbers; in case $Y$ is the unit disc the factors of automorphy for $(Y, H)$ with values in $G L(1, C)$ are of the form

$$
k(z, \phi)=c(\phi)\left(\frac{d \phi(z)}{d z}\right)^{m / M} h(\phi(z)) h^{-1}(z)
$$

where $h(z)$ is an entire nonvanishing function, $c(\phi)$ a suitable complex number, and $m$ and $M$ integers.

Suppose we are given a factor of automorphy $k$ for $(Y, H)$ with values in $C$. Suppose furthermore that $G$ acts holomorphically as a group of holomorphic automorphisms on a complex space $Z$, and the action of $g \in G$ on $z \in Z$ is denoted by $z g$. Then a holomorphic mapping $f: Y \rightarrow Z$ is called an automorphic function with values in $Z$ belonging to $k$ if

$$
f(\phi(y))=f(y) k(y, \phi)
$$

holds for every $\phi \in H$ and every $y \in Y$. Again there is a fiber bundle over $X$ with fiber $Z$ such that the set of cross-sections in it and the set of automorphic functions belonging to $k$ are in a bijective correspondence. This together with the theorems of $\$ 3$ assure the existence of automorphic functions in sufficiently general cases. By the same method one can investigate factors of automorphy depending holomorphically on parameters and automorphic functions depending on parameters. At the same time a deformation of the complex structure of $X$ can be dealt with.

\section{BIBLIOGRAPHY}

1. N. Akizuki and S. Nakano, Note on Kodaira-Spencer's proof of Lefschetz theorems, Proc. Japan. Acad. 30 (1954), 266-272.

2. M. F. Atiyah, Complex fibre bundles and ruled surfaces, Proc. London Math. Soc. 5 (1955), 407-434. 
3. - - Vector bundles over an elliptic curve, Proc. London Math. Soc. 7 (1957), $414-452$.

4. - Complex analytic connections in fibre bundles, Trans. Amer. Math. Soc. 85 (1957), 181-207.

5. - On the Krull-Schmidt theorem with application to sheaves, Bull. Soc. Math. France 84 (1956), 307-317.

6. H. Behnke and K. Stein, Entwicklung analytischer Funktionen auf Riemannschen Flächen, Math. Ann. 120 (1949), 430-461.

7. G. D. Birkhoff, $A$ theorem on matrices of analytic functions, Math. Ann. 74 (1913), 122-133.

8. - Infinite products of analytic matrices, Trans. Amer. Math. Soc. 17 (1916), 386-404.

9. - The generalized Riemann problem for linear differential equations...., Proc. Amer. Acad. Arts Sci. 49 (1913), 521-568.

10. — Equivalent singular points of ordinary linear differential equations, Math. Ann. 74 (1913), 134-139.

11. S. Bochner, A new viewpoint in differential geometry, Canad. J. Math. 3 (1951), 460-470.

12. A. Brill and M. Noether, Über die Entwicklung algebraischer Functionen und ihre Anwendung in der Geometrie, Math. Ann. 7 (1874), 269-310. 1950.

13. H. Cartan, Espaces fibrés analytiques complexes, pp. 1-9, Séminaire Bourbaki,

14. - Variétés analytiques complexes et cohomologie, pp. 41-55, Coll. de Bruxelles, 1953.

15. H. Grauert, Analytische Faserungen über holomorph-vollständigen Räumen, Math. Ann. 135 (1958), 263-273.

16. - Ein Theorem der analytischen Garbentheorie und die Modulräume komplexer Strukturen, Publ. Math. Inst. Hautes Etudes Sci. 5 (1960), 233-291.

17. H. Grauert and R. Remmert, Bilder und Urbilder analytischer Garben, Ann. of Math. 68 (1958), 393-443.

18. A. Grothendieck, Sur la classification des fibrés holomorphes sur la sphère de Riemann, Amer. J. Math. 79 (1957), 121-138.

19. R. C. Gunning, General factors of automorphy, Proc. Nat. Acad. Sci. USA 41 (1955), 496-498. 382.

20. - The structure of factors of automorphy, Amer. J. Math. 78 (1956), 357-

21. K. Hensel and W. Landsberg, Theorie der algebraischen Funktionen einer Variablen und ihre Anwendung auf algebraische Kurven und abelsche Integrale, Leipzig, 1902.

22. D. Hilbert, Über eine Anwendung der Integralgleichungen auf ein Problem der Funktionentheorie, Verh. d. III. Intern. Math. Kongr. Heidelberg 1904.

23. F. Hirzebruch, Über eine Klasse von einfach-zusammenhängenden komplexen Mannigfaltigkeiten, Math. Ann. 124 (1951), 77-86.

24. - Neue topologische Methoden in der algebraischen Geometrie, BerlinGöttingen-Heidelberg, 1956.

25. A. Hurwitz, Sur l'intégrale finie d'une fonction entière, Acta Math. 10 (1897), $285-312$.

26. K. Kodaira, Characteristic linear systems of complete continuous systems, Amer. J. Math. 78 (1956), 716-744.

27. - On Kähler varieties of restricted type, Ann. of Math. 60 (1954), 28-48. 
28. - On a differential-geometric method in the theory of analytic stacks, Proc. Nat. Acad. Sci. U.S.A. 39 (1953), 1268-1273.

29. K. Kodaira and D. C. Spencer, Divisor class groups on algebraic varieties, Proc. Nat. Acad. Sci. U.S.A. 39 (1953), 873-877.

30. - On deformations of complex analytic structures. I, II, Ann. of Math. 67 (1958), 328-466.

31. R. König, Riemannsche Funktionen- und Differentialsysteme in der Ebene, J. Reine Angew. Math. 148 (1918), 146-182.

32. J. L. Koszul, Multiplicateurs et classes caractéristiques, Trans. Amer. Math. Soc. 89 (1958), 256-266.

33. - Espaces fibrés associês et pre-associês, Nagoya Math. J. 15 (1959), $155-169$.

34. J. A. Lappo-Danilevsky, Mémoires sur la théorie des systèmes des équations différentielles linéaires, Chelsea, New York, 1953.

35. G. F. Mandžavidze and B. V. Hvedelidze, On the Riemann-Privalov problem with continuous coefficients, Dokl. Akad. Nauk SSSR 123 (1958), 791-794.

36. Y. Matsushima, Fibrés holomorphes sur un tore complex, Nagoya Math. J. 14 (1959), 1-24.

37. Th. Meis, Die minimale Blätterzahl der Konkretisierung einer kompakten Riemannschen Fläche, Schr. Math. Inst. Univ. Münster 16 (1960), 1-61.

38. L. M. Milne-Thomson, The calculus of finite differences, London, 1933.

39. A. Morimoto, Sur la classification des espaces fibrés vectoriels holomorphes sur un tore complexe admettant des connexions holomorphies, Nagoya Math. J. 15 (1959), $83-154$.

40. - Sur le groupe d'automorphismes d'un espace fibré principal analytique complexe, Nagoya Math. J. 13 (1958), 157-168.

41. J. Moser, The order of a singularity in Fuchs' theory, Math. Z. 72 (1960), 379398.

42. S. Murakami, Sur certains espaces fibrés principaux holomorphes admettant des connexions holomorphes, Osaka Math. J. 11 (1959), 43-62.

43. N. I. Mushelišvili, Singular integral equations, Groningen, 1953.

44. S. Nakano, On complex analytic vector bundles, J. Math. Soc. Japan 7 (1955), $1-12$.

45. - On a certain type of analytic fibre bundles, Proc. Japan Acad. 30 (1954), $542-547$.

46. - Parametrization of a family of bundles, Mem. Coll. Sci. Univ. Kyoto. Ser A 33 (1961), 353-366.

47. M. S. Narasimhan, Variations of complex structures on an open Riemann surface, Ann. Inst. Fourier, Grenoble 11 (1961), 493-514.

48. H. J. Nastold, Über Meromorphe Schnitte komplex-analytischerVektorraumbündel und Anwendungen auf Riemannsche Klassen. I, II, Math. Z. 69 (1958), 366-394; 70 (1958), 55-92.

49. N. E. Nörlund, Vorlesungen über Differenzenrechung, Berlin, 1924.

50. J. Plemelj, Riemannsche Functionenscharen mit gegebener Monodromiegruppe, Monatsh. Math. Phys. 19 (1908), 211-245.

51. H. E. Rauch, Weierstrass points, branch points, and moduli of Riemann surfaces, Comm. Pure Appl. Math. 12 (1959), 543-560.

52. B. Riemann, Zwei allgemeine Lehrsätze über lineäre Differentialgleichungen mit algebraischen Koeffizienten, pp. 357-369, Math. Werke, Dover, New York, 1953.

53. H. Röhrl, Über Differentialsysteme, welche aus multiplikativen Klassen mit 
exponentiellen Singularitäten entspringen. I, II, III, Math. Ann. 123 (1951), 53-75; 124 (1952), 187-218; 125 (1953), 448-466.

54. - Das Riemann-Hilbertsche Problem der Theorie der linearen Differentialgleichungen, Math. Ann. 133 (1957), 1-25.

55. - Einige Bemerkungen ïber komplex-analytische Vektorraumbïndel, Arch. Math. 8 (1957), 360-367.

56. - On holomorphic families of fiber bundles over the Riemannian sphere, Mem. Coll. Sci. Univ. Kyoto Ser. A 33 (1961), 435-477.

57. - Unbounded coverings of Riemann surfaces and extension of rings of meromorphic functions (to appear).

58. - - Über das Riemann-Privalovsche Randwertproblem (to appear).

59. - Difference equations and related functional equations (to appear).

60. J. P. Serre, Géométrie algébrique et géométrie analytique, Ann. Inst. Fourier, Grenoble 6 (1956), 1-42.

61. I. B. Simonenko, Riemann's boundary value problem with a continuous coefficient, Dokl. Akad. Nauk SSSR 124 (1959), 278-281.

62. N. Steenrod, The topology of fibre bundles, Princeton, 1951.

63. O. Teichmüller, Veränderliche Riemannsche Flächen, Deutsche Math. 7 (1944), 344-359.

64. C. Teleman, Sur les structures homographiques d'une surface de Riemann, Rev. Math. Pures Appl. 4 (1959), 295-303.

65. A. Weil, Généralisation des fonctions abéliennes, J. Math. Pures Appl. 17 (1938), $47-87$.

66. - Fibre spaces in algebraic geometry, Univ. of Chicago, 1952.

University of Minnesota, Institute of Technology, 